\title{
Emerging therapies for non-small cell lung cancer
}

\author{
Chao Zhang ${ }^{1,3}$, Natasha B. Leighl ${ }^{2}$, Yi-Long $\mathrm{Wu}^{1}$ and Wen-Zhao Zhong ${ }^{1 *}$
}

\begin{abstract}
Recent advances in the field of novel anticancer agents prolong patients' survival and show a promising future. Tyrosine kinase inhibitors and immunotherapy for lung cancer are the two major areas undergoing rapid development. Although increasing novel anticancer agents were innovated, how to translate and optimize these novel agents into clinical practice remains to be explored. Besides, toxicities and availability of these drugs in specific regions should also be considered during clinical determination. Herein, we summarize emerging agents including tyrosine kinase inhibitors, checkpoint inhibitors, and other potential immunotherapy such as chimeric antigen receptor $T$ cell for non-small cell lung cancer attempting to provide insights and perspectives of the future in anticancer treatment.
\end{abstract}

Keywords: Tyrosine kinase inhibitors, Checkpoint inhibitors, CAR-T, Bispecific antibodies, Lung cancer

\section{Background}

In the past few decades, systemic treatment for lung cancer remained to be cytoxicity agents with platinum-based regimens. ECOG1594 was the first trial comparing four different chemotherapy regimens for advanced non-small cell lung cancer (NSCLC) head to head [1]. All chemotherapy regimens showed almost the same efficacy with objective response rate (ORR) of $19 \%$ and 7.9 m median overall survival (OS). The platinum-based doublet chemotherapy seemed to reach the plateau since then. In 2005, the first-ever trial combining small molecular targeted agent known as bevacizumab, an anti-vascular endothelial growth factor (VEGF) monoclonal antibody, with doublet chemotherapy, had shown superiority of overall survival with this treatment modality in advanced non-squamous non-small cell lung cancer patients without brain metastasis [2]. Yet, several trials including molecular targeted agents and chemotherapy fail to reach the endpoints [3-5].

Epidermal growth factor receptor, a well-known biomarker for targeted therapy at present, was first brought up with potential clinical responsiveness to tyrosine kinase inhibitor gefitinib in 2004[6]. Since then the era of

\footnotetext{
* Correspondence: 13609777314@163.com

'Guangdong Lung Cancer Institute, Guangdong Provincial Key Laboratory of Translational Medicine in Lung Cancer, Guangdong Provincial People's

Hospital and Guangdong Academy of Medical Sciences, Guangzhou 510080,

Guangdong, People's Republic of China

Full list of author information is available at the end of the article
}

targeted therapy was uncovered and multiple trials demonstrated the efficacy of tyrosine kinase inhibitor (TKI) in oncogene-driven non-small cell lung cancer patients [711]. In these trials, significantly improved progression-free survival (PFS) was observed compared to tradition chemotherapy; however, no overall survival benefit was identified which may be partly due to high crossover rate after disease progression [12-14]. Moreover, resistance to tyrosine kinase inhibitor was inevitable and sequential treatment was warranted [7, 15-17].

Although up to $69 \%$ of patients with advanced NSCLC could harbor actionable driver mutations, a number of patients barely got a chance for more effective agents other than chemotherapy [16, 18-21]. Until 2013, immunotherapy was crowned as the first place of scientific breakthroughs [22]. Efficacy of immunotherapy for those without targetable oncogene mutation was proven from second-line treatment [23-27] to first-line treatment $[28,29]$. Through long-term follow-up, immunotherapy had also shown itself the greatest potential of long-term clinical benefit [30,31], even though the efficacy was not that satisfactory [23-30]. Indeed, similar to targeted therapy, patients may eventually develop resistance to immunotherapy [32, 33] and some may even suffer hyperprogression after immunotherapy [34,35]. The desire of novel agents that showed better efficacy, prolong survival benefit, and overcame resistance promoted the

(c) The Author(s). 2019 Open Access This article is distributed under the terms of the Creative Commons Attribution 4.0 International License (http://creativecommons.org/licenses/by/4.0/), which permits unrestricted use, distribution, and 
development of potential targets and corresponding drugs. In recent years, we have witnessed the birth of numerous emerging agents and their superior clinical responsiveness. Herein, we summarized the novel agents in tyrosine kinase inhibitors especially for epidermal growth factor receptor (EGFR) and anaplastic lymphoma kinase (ALK) inhibitors, checkpoint inhibitors, and other potential immunotherapy aiming to provide a landscape of emerging agents for NSCLC as well as insights and perspectives for the future in anticancer treatment.

\section{Epidermal growth factor receptor and human epidermal growth factor receptor 2 inhibitors Dacomitinib}

Dacomitinib is a selective and irreversible inhibitor for EGFR [36, 37]. In 2014, an official announcement from Pfizer indicated the trial failure of dacomitinib in patients with refractory advanced non-small cell lung cancer. However, based on superior results from phase II single-arm trial (ARCHER 1017) in the first-line setting, ARCHER1050, a phase III randomized control trial (ARCHER 1050) comparing dacomitinib and gefitinib head to head, was set to confirm its clinical efficacy and safety in expanded population. The results were promising, and the median PFS for dacomitinib and gefitinib was 14.7 months and 9.2 months, respectively ( $\mathrm{HR}=0.59,95 \%$ CI $0.47-0.74)$ [38]. Similar efficacy was shown between EGFR 19Del and EGFR 21L858R which suggested opposite results compared to the first-generation TKI in previous researches [39, 40]. Further OS results have been recently unleashed, and the median OS was $34.1 \mathrm{~m}$ with dacomitinib versus $26.8 \mathrm{~m}$ with gefitinib $(\mathrm{HR}=0.76,95 \% \mathrm{CI} 0.58-0.99)$ [41]. Higher incidence of adverse events compared to the first-generation TKI should be noticed [38, 41]. Currently, updated results of dose reduction in dacomitinib have been released and higher efficacy was found in dose modulation group [42]. Yet, efficacy results in patients with brain metastasis and resistant mechanism to dacomitinib were poorly explored and whether patients who had treatment failure after dacomitinib could still have a great chance of receiving osimertinib has not been answered [41]. Indeed, dacomitinib has been officially approved by the FDA in 2018 due to its superior performance in the first-line setting. Clinically, dacomitinib as a first-line treatment would be an optional choice, and hopefully, the third generation may be a salvage treatment after disease progression. But it would be too early to confirm its significant clinical role in first-line treatment neglecting the striking performance from osimertinib. Further clinical researches were warranted to provide evidence for a better therapeutic scheme.

\section{Osimertinib (AZD9291)}

Despite the high response rate to the first-generation TKI, majority of patients would suffer disease progression after
9-13 months of treatment [7,15-17]. The most common resistant mechanism to the first-generation TKI is p.Thr790Met point mutation (T790 M) with almost $60 \%$ $[15,20]$. Osimertinib, an irreversible third-generation TKI, was set to overcome resistance to T790M, and sensitive EGFR mutations (19Del and 21L858R) were covered as well $[43,44]$. In a phase II single-arm AURA2 study [44], ORR was 70\% (95\% CI 64-77) among 199 pretreated patients receiving osimertinib and manageable side effect was identified. Extension population-based AURA study showed that 201 pretreated patients harboring T790 M mutation received osimertinib with a median treatment duration of 13.2 months. Objective response rate was $62 \%$ ( $95 \%$ CI, $54 \%$ to $68 \%$ ), and median PFS was 12.3 months (95\% CI, 9.5 to 13.8) [45]. Treatment-related adverse events were milder compared to previous TKI [7-10, 44, $45]$. With the superior performance, the FDA has approved its indications in second-line treatment. To further demonstrate the efficacy of osimertinib in the first-line setting, FLAURA study has been put forward and preliminary results have been released. Osimertinib showed significantly prolonged PFS compared to standard EGFR-TKIs in first-line setting (18.9 months vs. 10.2 months, HR $=0.46,95 \%$ CI 0.37-0.57) [46]. So far, the median overall survival for both osimertinib and standard EGFR-TKI group was not reached. Favorable trend for osimertinib could be identified with a $P$ value of 0.007 . Indeed, compared to previous EGFR-TKIs, osimertinib revealed much longer PFS and better efficacy as well as decreased toxicity. And since that, the FDA has approved its first-line setting in the early 2018. Yet, should the winner take it all? Recent studies have provided more evidence and faith of using osimertinib in the first line. The exploratory postprogression outcomes of phase III FLAURA study has been reported showing not reached median second PFS in osimertinib arm while 20 months for standard of care EGFR-TKI arm [47]. Another study found that the continuation of osimertinib after disease progression could lead to a median second PFS of 12.6 months and be associated with longer overall survival compared with discontinuation [48]. Indeed, the mature results of OS are requested to further give a final deposition of this issue. The other focal aspect for osimertinib is the resistant mechanism. Till now, limited studies reported the resistant mechanism of osimertinib and extreme complicated resistant profiles were identified based on current data [49-51]. Fortunately, several preclinical and small size studies have provided potential treatment modalities to overcome the resistance, but an umbrella trial should be designed to address the pending issues [48, 52-63]. On the other hand, considering the rapid development of checkpoint inhibitors (CPIs) in advanced NSCLC, whether CPIs could benefit in patients with pan-negative oncogenes after osimertinib or treatment failure of novel 
combination modality remained to be explored in prospective trials. (Figure 1). Among patients with advanced lung cancer, brain metastasis was regarded as one of the major factors for poorer prognosis [64-66]. In contrast to the first-generation EGFR-TKI, osimertinib showed much better response rate in brain metastasis which may be due to higher penetration through the blood-brain barrier (BBB) $[67,68]$. Collectively, osimertinib would be a more competitive first-line treatment for advanced non-small cell lung cancer patients beyond the first-generation EGFR-TKIs and further OS data of FLAURA study was pending to decipher the order issue.

\section{AZD3759}

Over $50 \%$ of NSCLC patients with EGFR-activating mutations would develop CNS metastasis during treatment $[65,69,70]$. Poor survival was observed in these patients with 16 months for brain metastasis [64] and 4.5-11 months for leptomeningeal metastasis [66]. AZD3759 is an oral EGFR-TKI which was specifically designed to overcome the weak penetration of the blood-brain barrier [71, 72]. This drug contained no substrate for efflux transport [71] and achieved 100\% penetration through BBB [69], suggesting superior clinical efficacy in CNS metastasis. The BLOOM study is a phase I, open-label, multicenter trial evaluating the safety and preliminary antitumor efficacy of AZD3759 [69]. Tolerable safety profile was observed in this trial, and high consistent concentration of AZD3759 between CSF and free plasma was observed. However, whether a high concentration of AZD3759 in CSF would be translated into durable CNS response and not inferior efficacy in extracranial target lesions compared to previous EGFR-TKI warrants further clinical results.

\section{Poziotinib, TAK-788, afatinib, and pyrotinib}

In NSCLC, approximately $10-15 \%$ of patients harbored EGFR-activating mutations. For those whose tumor has sensitive EGFR mutation including deletion in exon 19 and mutation encoding p.L858R, standard first-generation TKI could probably provide dramatic efficacy [7-11]. However, approximately $10-12 \%$ of patients within have an in-frame insertion in exon 20 of their tumors [73-75]. The EGFR exon 20 insertions are generally resistant to most EGFR-TKIs $[76,77]$ which may be due to the altered drug-binding pocket of exon 20 [76]. Poziotinib has been proven to be a potent inhibitor of both EGFR and HER2 exon 20 insertion mutations through preclinical models and clinical experience [78]. Its preliminary clinical activity has been reported in 2018 WCLC with confirmed ORR of 43\% in advanced NSCLC [79]. Another novel agent for EGFR exon 20 insertion, TAK-788, has also been reported in 2018 WCLC [80]. The ORR was approximately $40 \%$ in NSCLC patients with EGFR exon 20 insertion. Remarkably, the disease control rate could reach up to $100 \%$ in this small group of population. Additionally, for HER2 mutations, afatinib has shown some activity through retrospective researches with limited prospective clinical

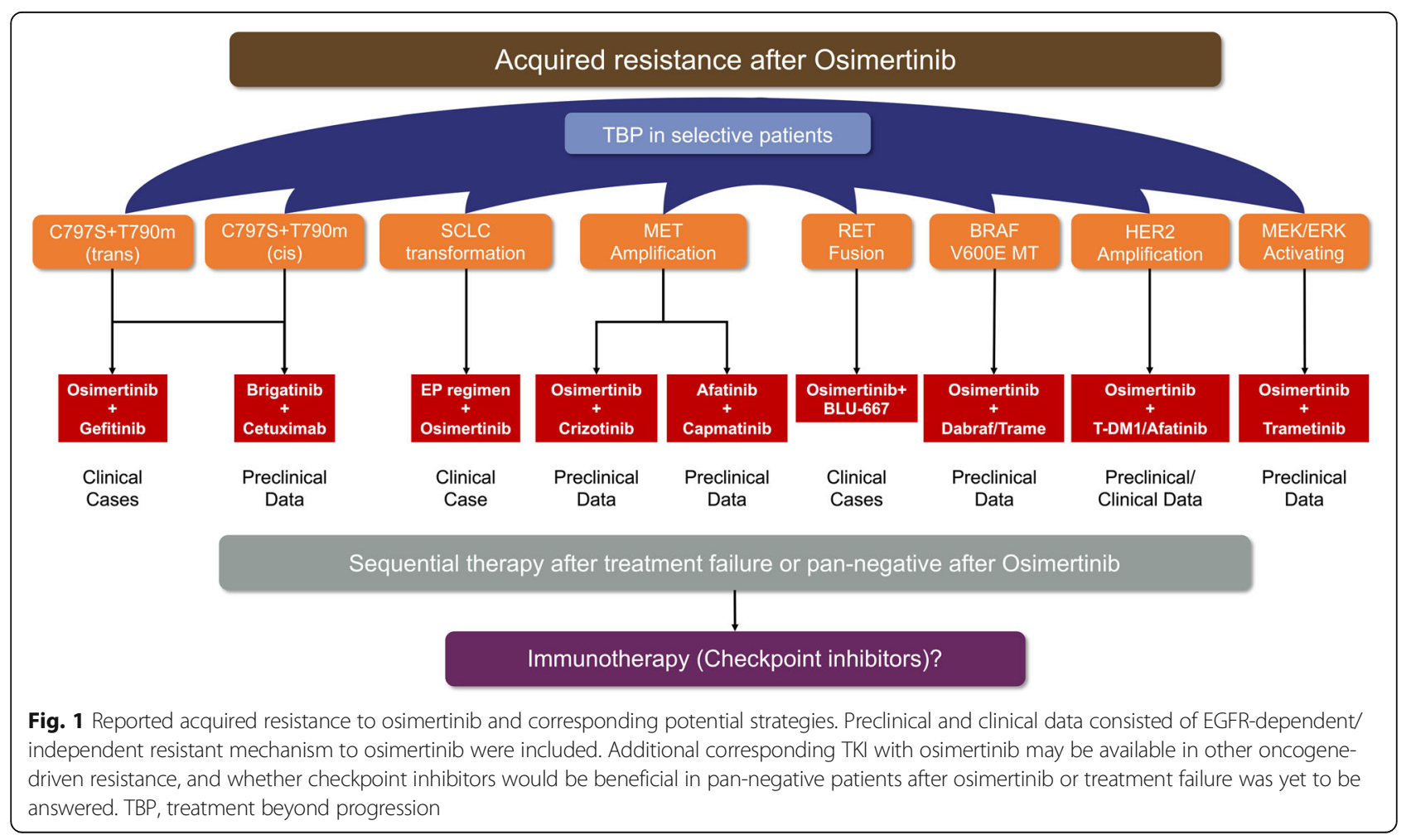


trials assessing its efficacy in HER2 mutation patients [8184]. A recent published phase II study (NICHE trial) showed a disease control rate of $53.8 \%$ with median PFS of 15.9 weeks and median OS of 56.0 weeks failing to verify the efficacy of afatinib in HER2 mutation patients [85]. On the other hand, pyrotinib is an oral, irreversible pan-HER receptor TKI. Preclinical data indicated effective antitumor activity in vitro and in vivo [86, 87]. A small sample size phase 2 study has investigated its efficacy and safety. Patients who received $400 \mathrm{mg}$ of pyrotinib showed an ORR of $53.3 \%$ and a median PFS of 6.4 months regardless of prior treatment lines along with tolerable adverse events [88]. Indeed, further larger sample size evaluation was warranted to verify its clinical value.

\section{Anaplastic lymphoma kinase and ROS1 proto-oncogene receptor kinase inhibitors Ceritinib}

The current standard first-line treatment for advanced non-small cell lung cancer harboring ALK rearrangement is crizotinib [89]. Despite the rapid response to crizotinib, most of the patients would suffer disease progression within 12 months [90, 91]. Approximately, a resistant mechanism in one third of patients with ALK-rearranged NSCLC was owning to ALK-dependent mutation including tyrosine kinase domain or amplification of ALK fusion. Ceritinib is a small molecule, oral tyrosine kinase inhibitor of ALK [92]. In contrast to crizotinib, ceritinib is 20 times more potent as crizotinib for ALK fusion, yet no tumor activity against MET was observed. In a phase I study evaluating the antitumor activity and safety in NSCLC harboring ALK fusion, ceritinib showed 58\% ORR in 114 patients with a dose of at least $400 \mathrm{mg}$. Median progression-free survival, although not that mature enough (38\% patients censored), was 7.0 months with a median follow-up time of 9.5 months [93]. Overall adverse events rate of grade 3 or 4 related to ceritinib therapy was $49 \%$ (varied from $20-80 \%$ among different dose groups), majority of which was gastrointestinal (GI) issues. Based on these superior clinical outcomes, the FDA granted an accelerated approval to ceritinib for the treatment in NSCLC patients harboring ALK rearrangement [94]. In 2016, the updated results of ASCEND-1 was released. Two hundred fifty-five patients who received at least 1 dose of ceritinib $750 \mathrm{mg} /$ day showed an overall response rate of $72 \%$ and $56 \%$ in treatment-naive and ALK inhibitor-pretreated groups, respectively. Intracranial disease control was reported in $79 \%$ of ALK inhibitor-naïve patients and $65 \%$ for ALK inhibitor-pretreated patients. However, approximately $81 \%$ of patients suffer at least 1 adverse events of grade 3 or 4 [95]. In a phase II trial (ASCEND-2), ceritinib was tested specifically in chemotherapy and ALK inhibitor-pretreated patients. ORR was $38.6 \%$ and median PFS was 5.7 months.
The intracranial overall response rate was $45.0 \%$ with similar and manageable tolerability as previous researches [96]. Moving into the first-line setting, ASCEND-4, a phase III study, showed $72.5 \%$ ORR and median PFS of 16.6 months. Yet, the control arm of this study was chemotherapy without providing head to head comparison to crizotinib. Besides, adverse events of grade 3 or 4 (78.0\%) were as high as previously reported with ceritinib $750 \mathrm{mg}$ daily [97]. Due to that, ASCEND-8, a phase I study assessing the tolerability of different dose of ceritinib in ALK-positive NSCLC, was initiated [98]. Compared to $750 \mathrm{mg}$ daily, $450 \mathrm{mg}$ with food may be optimal with favorable gastrointestinal tolerability. Further updated analysis suggested consistent efficacy between $450 \mathrm{mg}$ with food and $750 \mathrm{mg}$ along with less GI toxicities [99].

Attributed to a similar molecular structure with ALK fusion, ROS1 fusion may also be potential beneficiaries with ALK-TKI [100]. For advanced NSCLC patients harboring ROS1 fusion, crizotinib was first reported to have an antitumor activity for the treatment of ROS1 fusion. The ORR was $72.0 \%$ with a median PFS of 19.2 months. Toxicities were mild with no treatment related to adverse events of grade 4 or 5 [101]. Results were further demonstrated in a larger East Asian population in a phase II study. To be noticed, $13.4 \%$ of ROS1-positive patients within the study received a complete response to crizotinib [102]. In a phase II single-arm study, the efficacy and safety of ceritinib were assessed in a small sample size population harboring ROS1 fusion [103]. ORR was $62 \%$ with a median PFS of 24 months in overall patients who received at least 2 prior systemic treatment. Grade 3 or 4 toxicities of ceritinib $750 \mathrm{mg}$ daily in ROS1 fusion were much milder than in ALK fusion ( $37 \%$ vs. $~ 80 \%$ ) which may probably be owing to the diverse interaction between drugs and targets.

\section{Alectinib}

Similar to EGFR mutation, patients with ALK rearrangement would be under high risk of brain metastasis [104]. Alectinib is a highly selective inhibitor of anaplastic lymphoma kinase (ALK) which has shown both systemic and central nerve system efficacy in ALK-positive non-small cell lung cancer [70, 105-108]. J-ALEX trial is the first trial comparing alectinib and crizotinib as the first-line setting in advanced non-small cell lung cancer with ALK rearrangement, but only involving the Japanese population [109]. The result of median PFS was rather promising with 20.3 months for alectinib and 10.3 months for crizotinib. The superiority had been duplicated in the upcoming ALEX trial involving a larger amount of population [110]. According to the updated results, the median PFS for alectinib in first-line treatment was 34.8 months which was almost three times longer than the standard first-line treatment for ALK 
rearrangement [89]. Besides, the phase III ALUR study directly compared alectinib with chemotherapy in crizotinib-pretreated ALK-positive non-small cell lung cancer [111]. Median PFS was 9.6 months with alectinib and 1.4 months with chemotherapy as second-line treatment indicating an absolute clinical role of alectinib as a first-line setting. Besides, in contrast to other ALK-TKI such as crizotinib, alectinib did not have a substrate for efflux transport $[104,112,113]$ and penetration through $\mathrm{BBB}$ was significantly higher than crizotinib $[114,115]$. Recent data revealed high objective response rate of $73.3 \%$ with $100 \%$ central nerve system (CNS) disease control rate (DCR) in patients with ALK rearrangement and symptomatic or large CNS metastasis [115]. Although the resistant profile of crizotinib has been well described [90, 116, 117], little was known about alectinib and Gly1202Arg (G1202R) remained a hot potato for alectinib $[118,119]$. Indeed, considering its tremendous improvement in first-line treatment compared to other ALK TKIs, alectinib should currently be the first option from all aspects for treatment-naive patients with advanced non-small cell lung cancer harboring ALK rearrangement.

\section{Brigatinib (AP26113)}

Brigatinib, another second-generation highly potent ALK-TKI, was also designed for a broad range of ALK resistance mutations [120]. Similar to lorlatinib, brigatinib was proved to be efficiently inhibiting all clinically relevant ALK resistance mutations including ALK G1202R through preclinical models [121]. However, another study showed diverse outcome in preclinical models with IC50 of $129.5 \mathrm{nM}$ to brigatinib indicating inferior sensitivity to G1202R [106]. In a multicenter retrospective study, one alectinib-pretreated patient harboring G1202R had a progressive disease as the best response to brigatinib [122]. Whether brigatinib could overcome the resistance to G1202R remained to be explored in a larger sample size trial. Through a phase II trial of brigatinib in patients with crizotinib-refractory ALK-positive NSCLC, brigatinib yielded both substantial systemic and intracranial response and $180 \mathrm{mg}$ once daily was proven to have better efficacy with acceptable safety [123]. Currently, an interim analysis of ALTA-L1 has been reported, showing $51 \%$ of progression risk reduced although median PFS was not reached [124]. Although, relevant research suggested the brain accumulation of brigatinib may be restricted by P-glycoprotein (P-gp) and breast cancer resistance protein (BCRP) [125]. For patients with brain metastasis at baseline in ALTA trial, brigatinib achieved potent efficacy with a progression risk reduction of $73 \%$. Similar adverse events were identified compared to previous ALK-TKI [89, 97, 126-128]. Most importantly, the final PFS and OS result of brigatinib should be expected to decipher whether brigatinib might be superior to the new standard first-line alectinib.

\section{Lorlatinib}

Lorlatinib is a highly potent and brain-penetrant third-generation ALK-TKI in patients with advanced ALK-positive NSCLC [106]. Most ALK-positive patients treated with first- or second-generation ALK-TKI would develop resistance to TKI including ALK Gly1202Arg (G1202R) solvent-front mutation located at the solvent-front region of ALK, and can impair drug binding through steric hindrance $[106,129]$. A preclinical data showed lorlatinib was the only ALK inhibitor to potently inhibit wide-range ALK secondary mutations, including ALK G1202R [130]. So far for lorlatinib, only phase 1 and 2 study has been released, and the results were promising [131]. For treatment-naive patients, ORR was $90 \%$ and $69.5 \%$ for crizotinib-treated patients. Based on a preliminary analysis of paired cerebrospinal fluid and plasma samples, lorlatinib has been demonstrated with a high degree of penetration across the blood-brain barrier [104]. In this phase 1 and 2 trial, the intracranial response for treatment-naive patients was $66.7 \%$ while $87 \%$ for crizotinib-treated patients. Additionally, a phase I study evaluating the efficacy and safety of lorlatinib in ALK/ROS1-positive NSCLC showed encouraging results in either ALK rearrangement or ROS1 rearrangement patients regardless of treatment lines [132]. For ALK-positive patients, the overall ORR was $46 \%$ along with a median PFS of 9.6 months and 50\% along with 7.0 months for ROS1positive patients. Collectively, although PFS result was not that mature enough, considering its wide range profile for resistance to ALK-TKI, lorlatinib would be an optional sequential treatment for patients previously treated with ALK-TKI. A phase III study was being investigated comparing lorlatinib to crizotinib at first-line setting (NCT03052608), and the preliminary results may be presented in 2020 .

\section{Ensartinib (X-396)}

Ensartinib (X-396) is a novel, aminopyridazine-based small molecule drug that could potently inhibit ALK. Through preclinical study, tenfold more potent than crizotinib inhibiting ALK-positive lung cancer cell lines was observed [133]. Results of a multicenter expansion study had been first reported in 2016 WCLC [134] showing similar response rate and adverse events compared to previous ALK-TKI. Currently, this first-in-human phase I/II multicenter study has revealed the survival benefit of ensartinib with median PFS of 26 months in treatment-naive patients and 9 months for crizotinib-pretreated patients [135]. ORR for treatment-naive patients was $80 \%$ and $69 \%$ for previously treated patients. Among patients with brain metastasis, intracranial disease control rate could reach up to 92.9\%. eXalt3 is a phase 3 randomized trial comparing ensartinib and crizotinib head to head [136]. The preliminary results would be released in 2019 which would further verify and discuss its clinical role in ALK-positive lung cancer patients (Table 1). 
Chang et al. Journal of Hematology \& Oncology

(2019) 12:45

Page 6 of 24

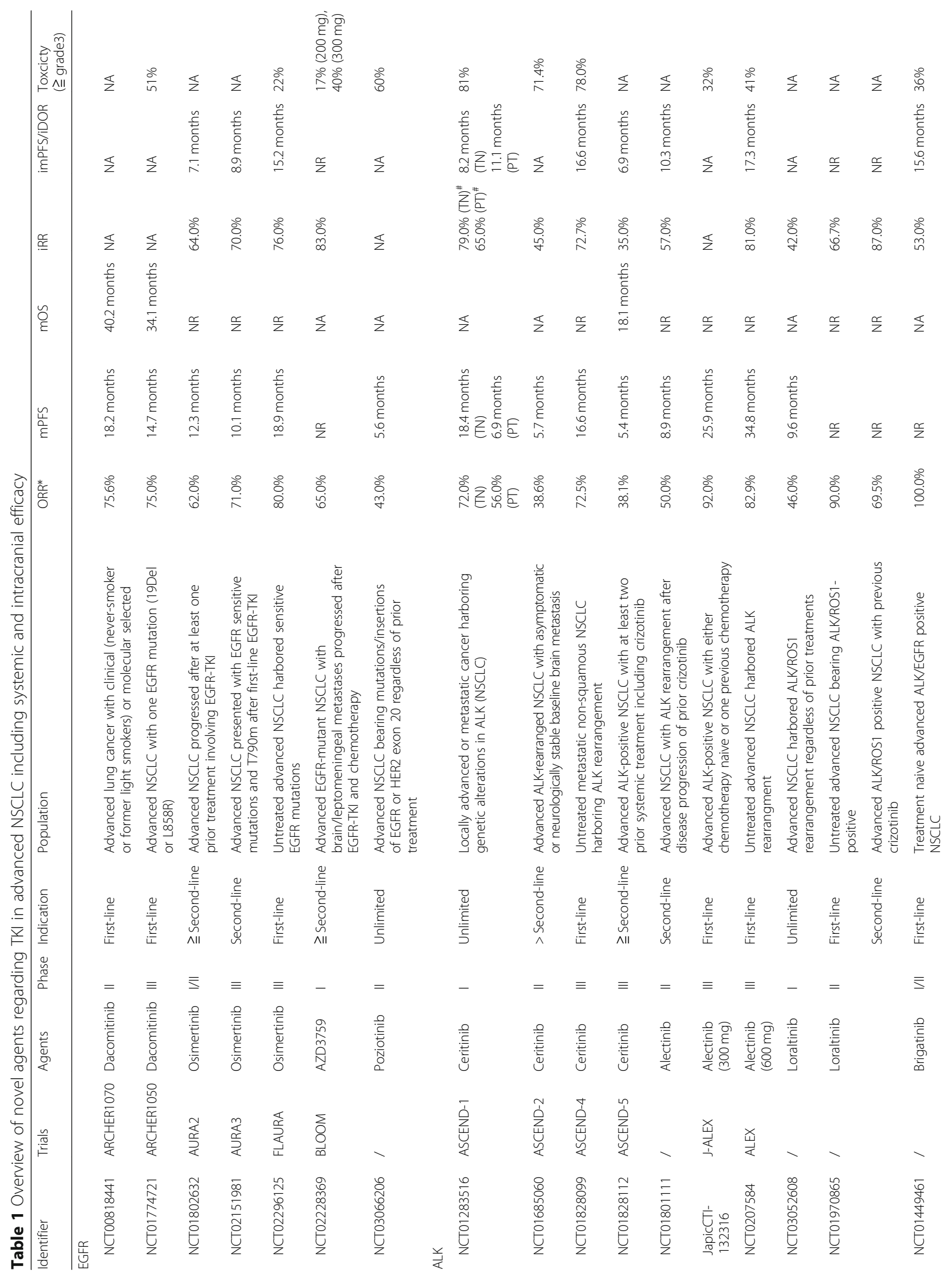




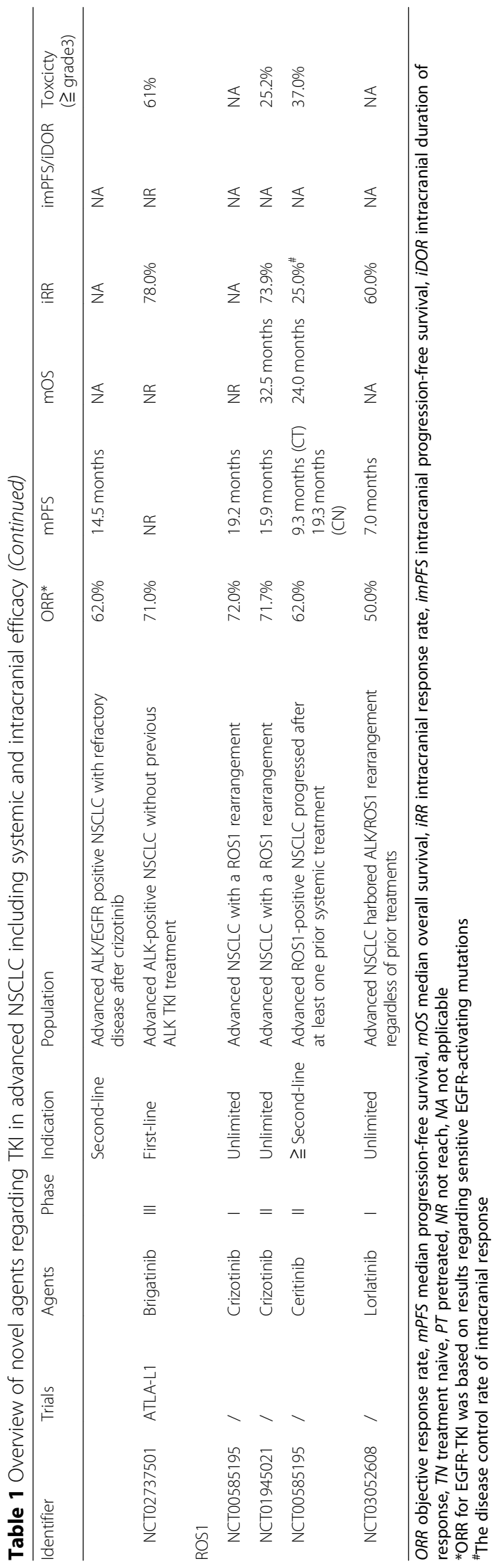


Other novel tyrosine kinase inhibitor

Entrectinib (RXDX-101), larotrectinib (LOXO-101), and LOXO195

Recurrent gene fusions are one of the essential oncogenic drivers to promote tumor growth among varied malignancies [137]. Similar to ALK and ROS1 rearrangement, fusions of NTRK1, NTRK2, and NTRK3 are actionable drivers of tumor growth. The incidence of NTRK fusion in solid tumor was reported as $0.1 \%$ [138]. Entrectinib and larotrectinib were both inhibitors targeting NTRK fusions [139, 140]. Unlike larotrectinib, entrectinib also showed efficacy in ROS1 and ALK rearrangement [139]. Two phase I study (ALKA-372-001 and STARTRK-1) assessing entrectinib in NTRK-, ROS1-, and ALK-positive solid tumor showed promising efficacy and durable clinical benefit of 32 months in a ROS1-positive lung cancer patients [139]. Besides, promising intracranial efficacy was observed indicating high penetration through BBB.

Although limited data of larotrectinib was shown, a recent study containing three phase I/II clinical trials was published and splendid responsiveness was revealed in pan-solid tumor including lung cancer harboring NTRK fusion [140]. Responsiveness was observed regardless of tumor type, and the overall response rate was $80 \%$. Based on its superb outcome, the FDA has approved its application in patients of solid malignancies harboring NTRK fusion. Despite the preliminary clinical results, primary and acquired resistance has already been characterized in several studies [141, 142]. To overcome the resistance mediated by acquired kinase domain mutations, LOXO-195, a selective TRK-TKI, was designed and preclinically proven to be highly potent in vitro [143]. Two patients whose tumor developed an acquired resistance to larotrectinib were treated with LOXO-195 and showed potential efficacy, but relevant data was warranted specifically in lung cancer patients for the future.

\section{Repotrectinib (TPX-0005)}

Similar to entrectinib and larotrectinib, repotrectinib is a next-generation TKI developed to inhibit clinically recalcitrant solvent front substitutions involving TRK, ROS1, and ALK. In a preclinical study, among common the acquired resistance to ALK, ROS1, and TRK including ALK G1202R, ROS1 G2032R, and TRKB G639R, repotrectinib showed high efficacy in vitro compared to other ALK/ROS1/TRK inhibitors. For patients with brain metastasis, a significant clinical challenge, this next-generation TKI showed superior efficacy compared to crizotinib in patients with CNS metastasis. This may be partly due to its smaller molecule structure compared to previous TKI drugs [144]. The current phase I/II clinical trial investigating the efficacy and safety of repotrectinib is still ongoing (NCT03093116), and further results should be expected.

\section{RXDX-105, LOXO-292, and BLU-667}

RET fusion is a well-established driver oncogene in a variety of malignancies. In lung cancer, RET fusion was found in 1-2\% unselected cases [145]. RXDX-105 is an orally, VEGFR-sparing, multikinase inhibitor with activity against RET. Compared to other RET inhibitors including cabozantinib, vandetanib, and lenvatinib, RXDX-105 showed high preclinical activity [146-149]. In a phase I/Ib trial [150], treatment-naive NSCLC patients with RET fusion showed 19\% ORR to RXDX-105 while $0 \%$ ORR for TKI-pretreated patients. Specifically looking into different upstream partners for RET, only non-KIF5B RET fusion showed satisfactory clinical efficacy to RXDX-105 which is similar to other RET inhibitors.

Unlike multikinase inhibitors such as RXDX-105 which may be under substantial "off-targets" hindering their clinical efficacy, LOXO-292 is a novel RET inhibitor with high selectivity [147, 149, 151, 152]. Through preclinical research and clinical experience with LOXO-292, it showed both high selectivity and responsiveness to RET fusion cell lines. Besides, LOXO-292 revealed high efficacy in KIF5B-RET fusion engineered cells which were different from previous RET inhibitors [151]. Another similar small molecule specifically targeting RET is BLU-667 which covered both RET fusion and RET-activating mutations as well [153]. Compared to RXDX-105, cabozantinib, and vandetanib, BLU-667 showed a broad range of efficacy in RET fusion and activating mutation with high selectivity in KIF5B-RET preclinically. A phase I, first-in-human study (NCT03037385) which tried to define the maximum tolerated dose and evaluate the safety along with antitumor activity is still ongoing, and clinical potentials of such high selective RET inhibitors will be elucidated in the future.

\section{Capmatinib (INC280)}

Capmatinib is a highly potent MET inhibitor [154], and its single-agent activity has been observed in preclinical models with strong MET amplification, overexpression, and mutations. MET amplification could be accounted for $5-26 \%$ in patients resistant to previous EGFR-TKI [20, 155-159]. Preclinical research suggested INC280 could restore sensitivity to erlotinib and promote apoptosis in EGFR-mutant NSCLC models [160]. As a clinical rationale for the combination of capmatinib and EGFR-TKI, a phase Ib/II single-arm trial evaluated the combination of INC280 and gefitinib in EGFR-TKI-pretreated patients [161]. Overall response rate across phase Ib/II regardless of MET copy number was $27 \%$. In patients with high MET amplification 
(copy number $\geq 6$ ), the ORR was $47 \%$ with acceptable adverse events. However, the survival data was not mature enough and the resistant mechanism of capmatinib has not been released yet. A study established MET-amplified NSCLC cell lines which showed an acquired resistance to capmantinib. With further examination, they found that the combined treatment of EGFR or PIK3CA would dramatically suppress cell proliferation and downstream signals [162]. This may partly suggest an alternative therapeutic strategy to overcome the resistance to capmatinib, but further clinical researches were required to elucidate.

\section{Dabrafenib and trametinib}

BRAF mutations occurred in about $2-4 \%$ of lung adenocarcinoma, and approximately $50 \%$ of them were BRAF V600E mutations $[163,164]$. BRAF V600E mutations were reported to have shorter overall survival, and limited patients responded to chemotherapy compared to wild-type BRAF $[165,166]$. Vemurafenib was the first BRAF V600E inhibitor assessed in a basket trial which indicated a $42 \%$ overall response rate within BRAF V600E mutation NSCLC [167]. Dabrafenib was a highly potent adenosine triphosphate-competitive inhibitor of BRAF kinase selective for the BRAF V600E mutations [168]. In a phase II non-randomized trial, the disease control rate (DCR) was $53 \%$ with a median PFS of 5.5 months. Serious adverse events were reported in $42 \%$ of patients [169]. Through preclinical study, dabrafenib plus trametinib had shown high antitumor activity in BRAF V600E mutation cell lines, and clinically, BRAF plus MEK inhibitors revealed improved clinical outcome in patients with BRAF V600E mutant metastatic melanoma [170, 171]. In two phase II non-randomized trials, consistent overall response rate was observed with $63.2 \%$ and $64.0 \%$ in previously treated and untreated patients, respectively. Similar median PFS was found as well in previously treated and untreated patients with 8.6 months and 10.9 months, respectively [172, 173]. Within previously treated patients, grades 3 and 4 events occurred in $49 \%$ of patients while almost $73 \%$ for untreated patients. So far, considering limited choices in BRAF mutation especially V600E mutations, dabrafenib plus trametinib should be the first option in these group of patients.

\section{Anlotinib}

Anlotinib is a novel, small molecule receptor tyrosine kinases (RTKs) and inhibits both tumor proliferation and angiogenesis [174-176]. Preclinical studies have shown that anlotinib has emerged much stronger anti-angiogenic activity than other anti-angiogenesis agents [177]. Clinically, the efficacy and safety of anlotinib was first demonstrated in a randomized phase II study as a third-line therapy in advanced NSCLC [178]. Patients in the anlotinib group showed a significantly longer PFS than the placebo group (4.8 months vs. 1.2 months). Although no statistical significance was shown in OS, favor trend of survival benefit was identified in the anlotinib group (9.3 months vs. 6.3 months). Final results of an expanded population phase III randomized trial (ALTER 0303) has been released last year in ASCO meeting and showed both prolonged PFS and OS in the anlotinib group with well-tolerable adverse events indicating anlotinib as a potential third-line treatment in advanced NSCLC patients.

\section{Checkpoint inhibitors \\ Pembrolizumab, nivolumab, and atezolizumab}

With the rapid growth of immunotherapy these years, tradition chemotherapy in pan-negative advanced non-small cell lung cancer has been challenged from second-line treatment to first-line treatment by single-agent checkpoint inhibitors [23, 24, 27, 28]. For checkpoint inhibitors, the expression of PD-L1 has been considered as a major predictive factor for immunotherapy so far [179]. Given that significant discrepancy results of Keynote-024 and Checkmate-026[29, 180], only highly selective patients should be available for a single agent in the first-line setting. Back to the era of targeted therapy, combination strategies have achieved great success, and theoretically, this may probably work out in checkpoint inhibitors [181-183]. Keynote-021 first reported preliminary results of checkpoint inhibitors combined with chemotherapy [180]. Superior response rate and progression-free survival were observed with minor increased toxicities. Identical results were duplicated in phase III study Keynote-189 and Keynote-407 for lung adenocarcinoma and squamous cell carcinoma, respectively $[184,185]$. To be noticed, the component of combination immunotherapy seemed to significantly influence the incidence of adverse events. Cisplatin or paclitaxel showed much better tolerance than carboplatin or nab-paclitaxel as combination components. In Keynote-042 (2018 ASCO meeting), single-agent pembrolizumab has broadened its indication to a larger population with PD-L1 positive. Yet, patients with high expression PD-L1 in both trials showed a similar clinical outcome. Considering the cost-effectiveness and toxicities, it would be optimal to provide single-agent pembrolizumab in patients with PD-L1 high expression while combination regimens for low or negative PD-L1 expression. As for nivolumab, even post hoc analysis with stratification of tumor mutation burden showed statistical significance, and it is still a negative trial showing no significant improvement between single-agent nivolumab and platinum-based chemotherapy in PD-L1-positive patients based on the study design probably due to high crossover rate and non-highly selective patients. Checkmate-227 was the first reported 
combination trial involving nivolumab in advanced NSCLC. Compared to standard platinum-based chemotherapy, nivolumab plus ipilimumab revealed a significantly improved ORR (45.3\% vs. $26.9 \%)$ and prolonged mPFS (7.2 months vs. 5.5 months) in patients with high mutation burden regardless of PD-L1 expression [186]. Indeed, OS was not mature enough to present preliminary data and comparison between other arms as well as subgroup analysis was not released yet. Additionally, the three phase III trials of combination regimens involving atezolizumab (IMpower 150, IMpower131(2018 ASCO meeting), IMpower132(2018 WCLC meeting)) have all shown superiority in clinical outcome with tolerable adverse events in either non-squamous or squamous NSCLC [187]. Details of all posted trials with combination regimens are summarized in Table 2.

\section{Avelumab}

Avelumab is a fully human immunoglobulin G1 (IgG1) monoclonal antibody [188]. Beyond pembrolizumab, nivolumab, and atezolizumab, it is one of the last PD-L1 inhibitors along with durvalumab to access the market. Avelumab had been first approved in the USA for the treatment of metastatic Merkel cell carcinoma. In contrary to other PD-1/PD-L1 drugs, the binding of avelumab to the surface of tumor cell via PD-L1 could induce natural killer cell-mediated antibody-dependent cellular cytotoxicity (ADCC) which may enhance its clinical efficacy $[189,190]$. In a phase Ib, multicenter trial (JAVELIN Solid Tumor), patients with advanced, platinum-treated NSCLC were given a single-agent avelumab [188]. Acceptable safety profile was observed, and $50 \%$ of patients achieved disease control. Similar median progression-free survival and overall survival compared to previous PD-1/PD-L1 were observed with 17.6 weeks and 8.4 months, respectively [23, 26, 27]. Clinical efficacy was consistent with the level of PD-L1 expression, and higher expression of PD-L1 may be translated into a longer survival benefit. Recent results from a randomized phase 3 trial (JAVELIN Lung 200) also investigating the efficacy and safety of avelumab in platinum-treated patients with advanced NSCLC have been released [191]. In PD-L1-positive ( $\geq 1 \%$ ) patients, no significant survival benefit was observed between the avelumab and docetaxel groups (11.4 months vs. 10.3 months) except the high PD-L1 expression groups ( $\geq 50 \%$ cutoff and $\geq 80 \%$ cutoff). Increased ORR was consistent with the higher expression of PD-L1 in avelumab group instead of docetaxel group indicating PD-L1 as an essential predictive biomarker for avelumab. However, according to the primary endpoint this trial set up initially, it is a negative study even with numerical significance in survival. Other relevant trials including JAVELIN Lung 100, JAVELIN Lung 101, and JAVELIN Medley were still ongoing
(Table 3), and the closest report of JAVELIN Lung 100 will be released in 2019 .

\section{Durvalumab}

Durvalumab is a selective, high affinity human IgG1 monoclonal antibody which impedes PD-L1 from binding to PD-1 and CD 80 [192, 193]. Its clinical efficacy was first reported at the 2014 ASCO meeting through a phase I study, showing limited toxicity and potential response rate. On account of previous single-agent checkpoint inhibitor success in second-line treatment $[23,24$, 26 , 27], several phase trials of combination regimens involving durvalumab were initiated [194, 195]. Preliminary results of these studies showed no significant difference to other checkpoint inhibitors. One should notice that combination regimens involving durvalumab in both TATTON and CAURAL study revealed an extremely high risk of developing interstitial pneumonia, leading to the termination of both trials. The phase II ATLANTIC trial (NCT02087423) evaluated the efficacy of durvalumab as a third-line treatment in advanced NSCLC [196]. The ORR was $7.5 \%, 16.4 \%$, and $30.9 \%$ in patients with PD-L1 expression of $<25 \%,>25 \%$ and $>$ $90 \%$, respectively. PFS in patients with high PD-L1 and low/negative PD-L1 expression was 3.3 and 1.9 month. To be noticed, cohort 1 in this trial receiving single-agent durvalumab included advanced NSCLC patients with EGFR-sensitive mutations or ALK rearrangement. The ORR in this group was not remarkable even in PD-L1 high expression population. Two well-known phase III trials with diverse ending were unleashed last year. The MYSTIC trial (NCT02453282) assessing durvalumab plus tremelimumab or durvalumab monotherapy versus platinum-based chemotherapy showed both combination and single-agent regimen which failed to reach the primary endpoint without PFS benefit. The PACIFIC trial (NCT02125461), on the other hand, achieved great success and led to a treatment paradigm shift for unresectable locally advanced NSCLC [197, 198]. Additionally, several phase III trials of durvalumab are pending or ongoing, and hopefully, more optional treatment would be provided (Table 4).

\section{Potential novel treatment modalities for lung cancer Chimeric antigen receptor $T$ cell and bispecific antibodies} Beyond the field of checkpoint inhibitors, another immunotherapy such as adoptive cellular immunotherapy has emerged as a remarkable treatment modality in the past decades [199, 200]. Unlike checkpoint inhibitors, which induce antitumor activity through blocking the barrier between effective $\mathrm{T}$ cells and tumor cells [201-203], adoptive cellular immunotherapy is a novel approach providing "artificial" effective $\mathrm{T}$ cells to specifically target tumor cells directly regardless of tumor types [204-206]. With 


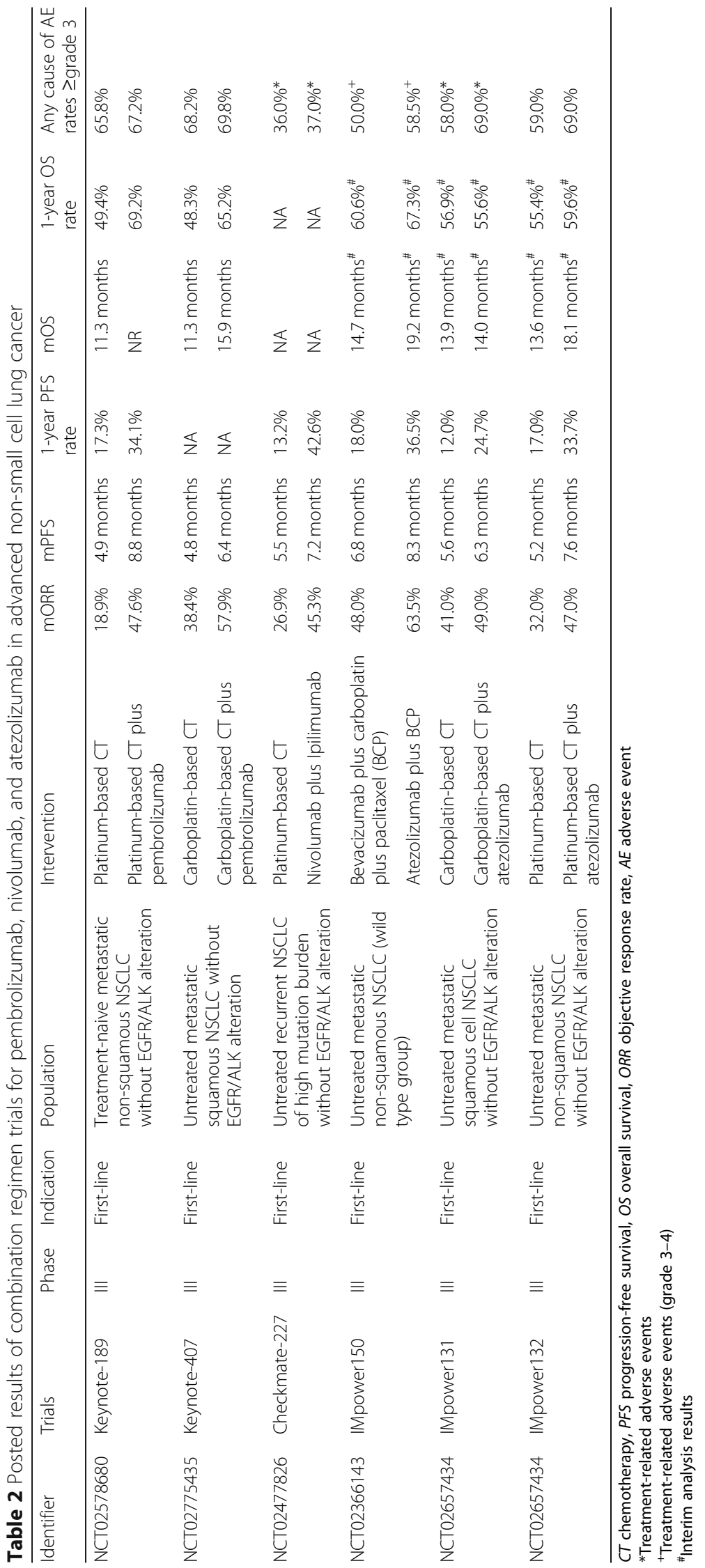




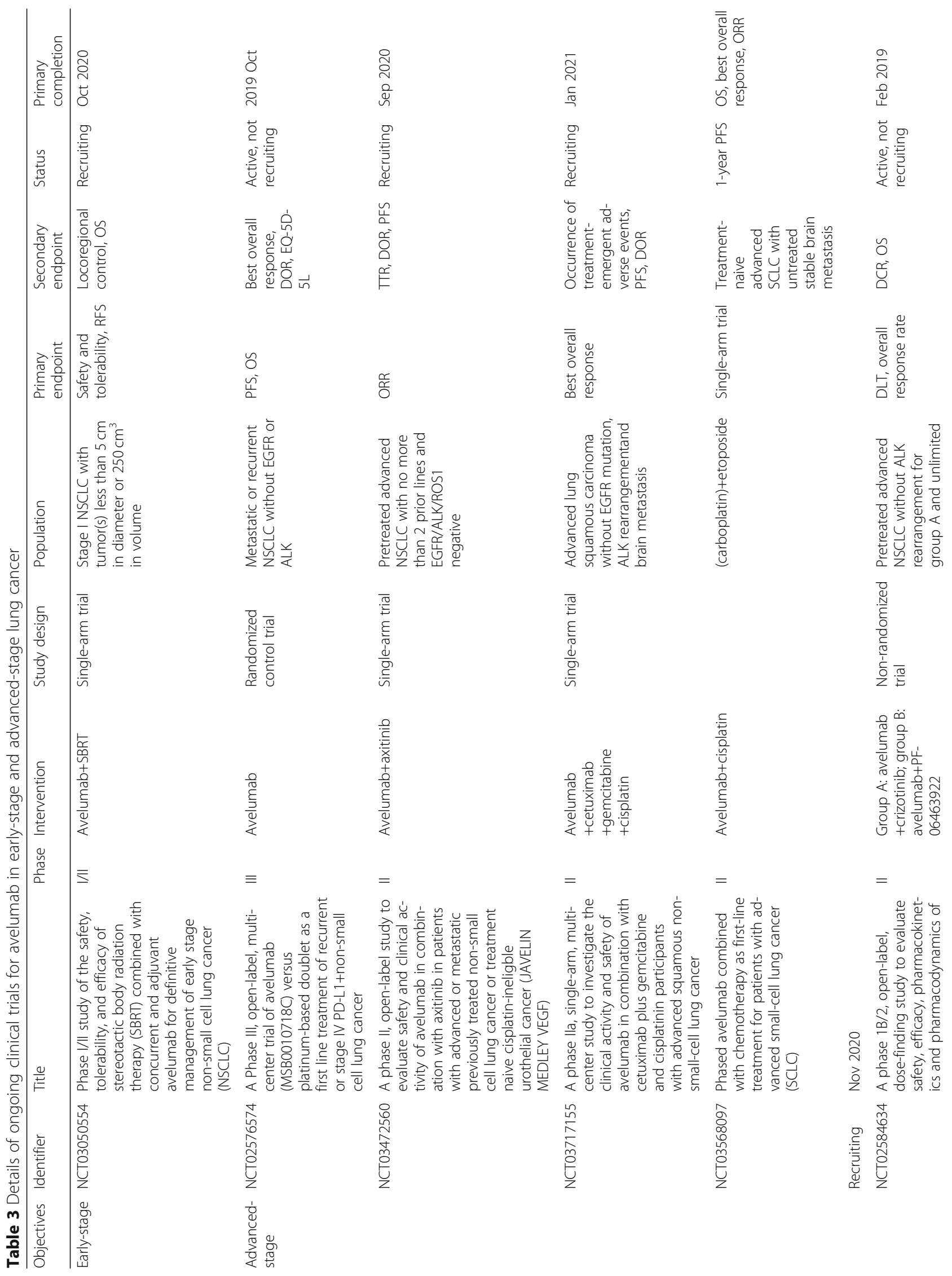




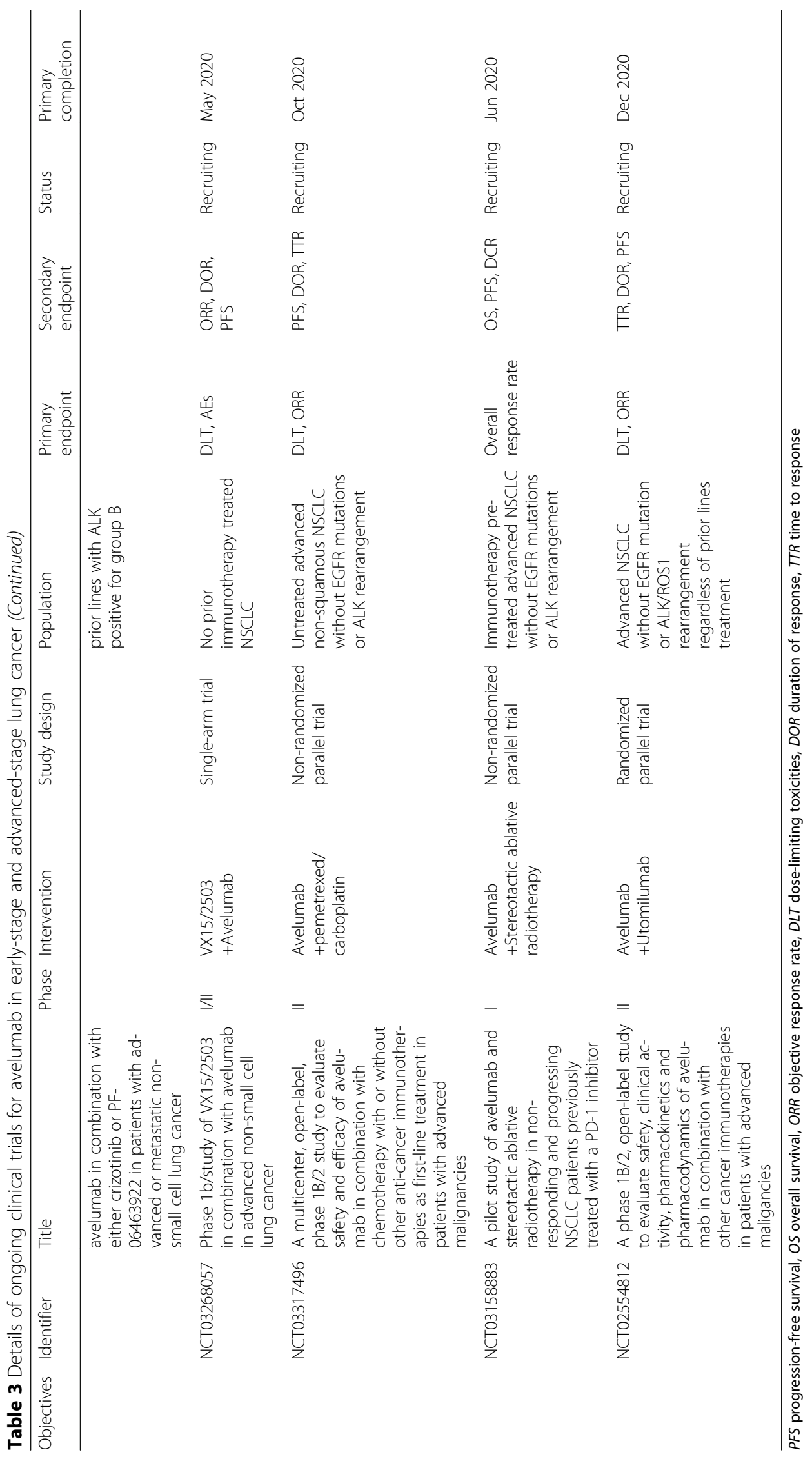




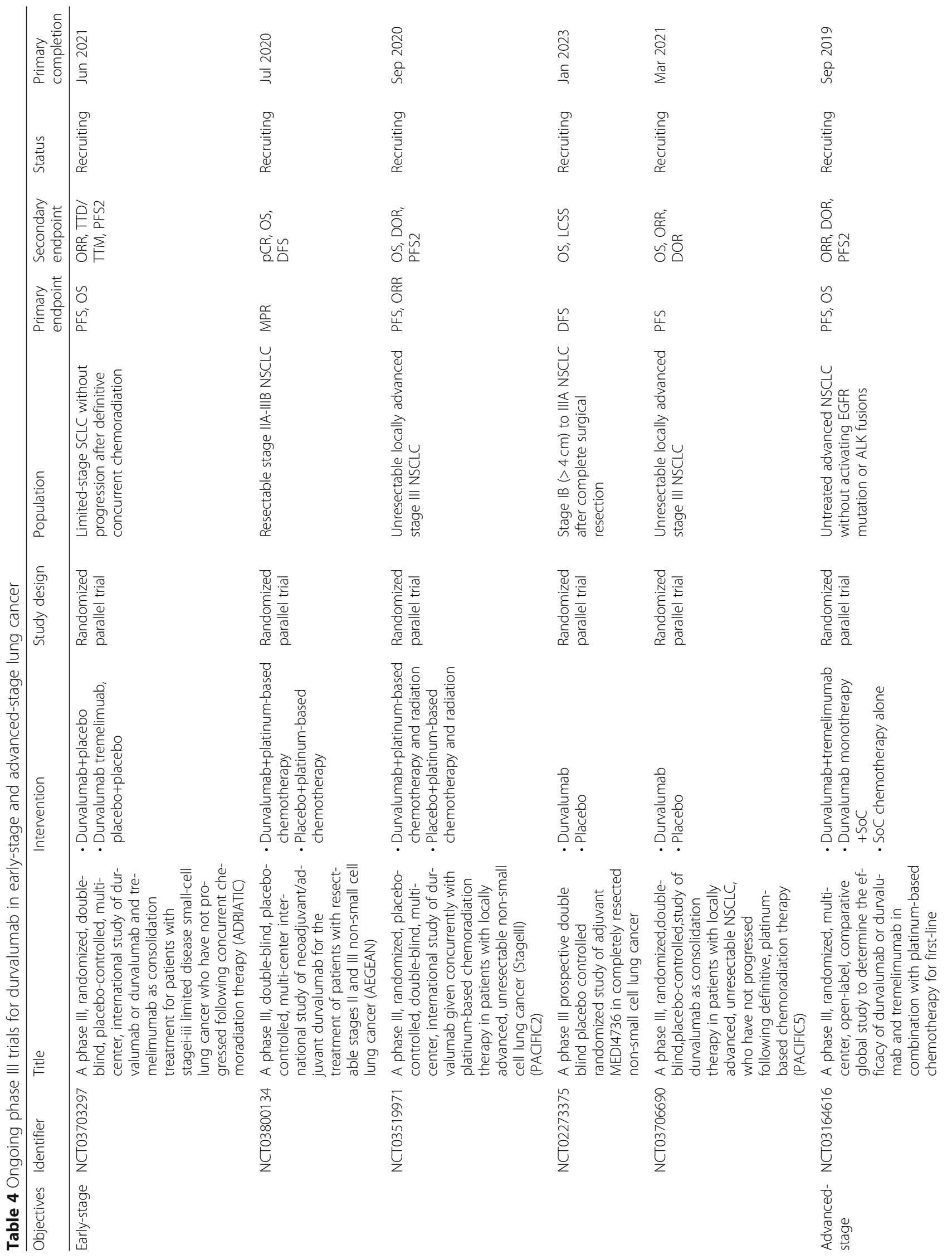




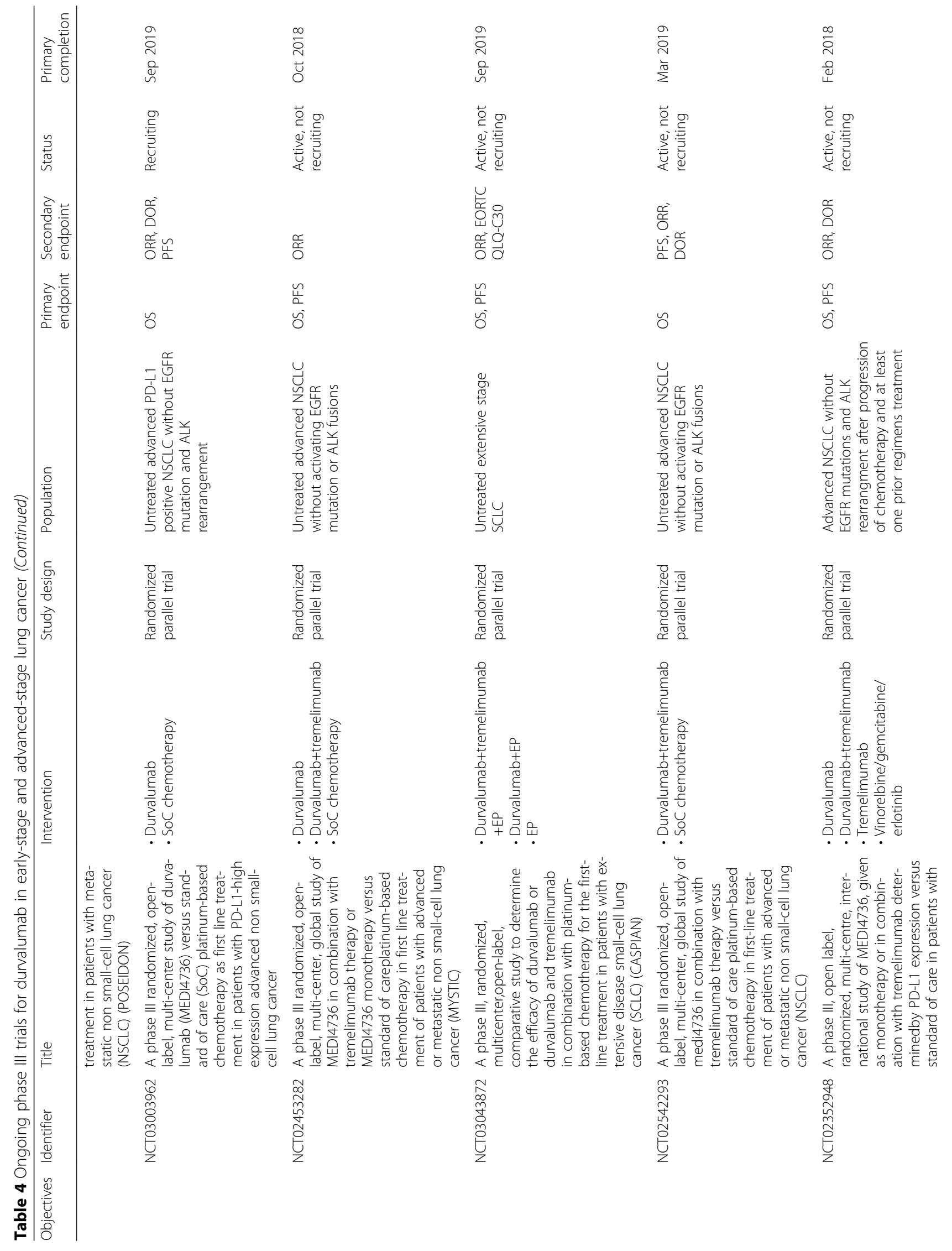




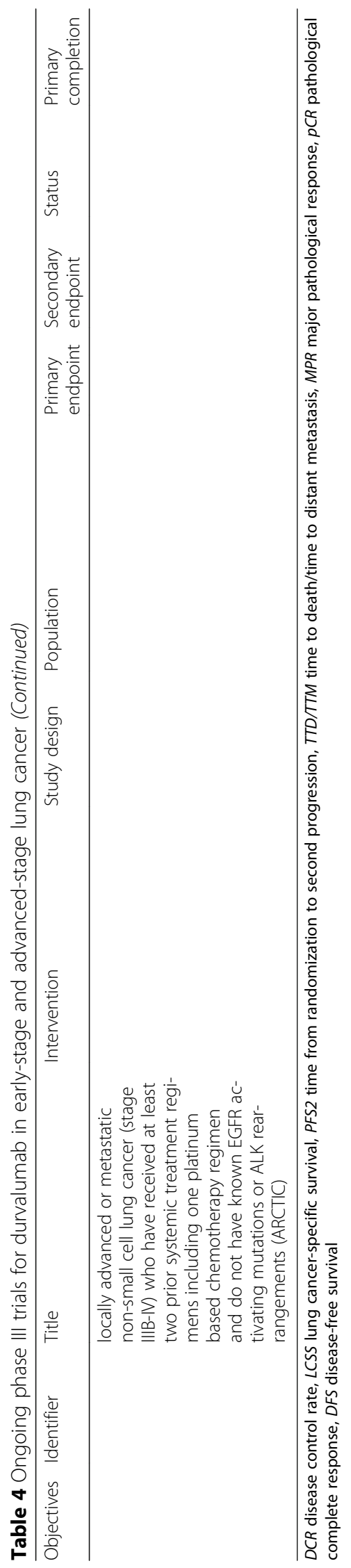


that being said, adoptive cellular immunotherapy may bring broad range effect on different tumors compared to checkpoint inhibitors, which have been reported diverse response among a variety of tumors [207-213]. So far, significant advances in chimeric antigen receptor $T$ cell (CAR-T) have been accelerated in hematological malignancies especially for CD19-targeted CAR-T-cell therapy in leukemia [214218]. However, in solid tumors, it is tough to design CAR-T because no such surface antigen as unique as CD19 has yet been identified [219]. Indeed, numerous clinical trials of CAR-T regarding lung cancer have been initiated including tumor-associated antigen (TAA) of EGFR (NCT02862028, NCT01869166), HER2 (NCT00889954, NCT01935843), carcinoembryonic antigen (CEA) (NCT01723306, NCT02 349724), and mesothelin (MSLN) (NCT01583686, NCT03054298). Gladly, several trials focusing on NSCLC were initiated and ongoing in China [220]. One clinical trial of EGFR-specific CAR-T regarding non-small cell lung cancer (NCT01869166) had reported its preliminary results. $45.5 \%$ (5/11) of advanced NSCLC patients achieved stable disease, and 2 achieved partial response (PR). Treatmentrelated adverse events were manageable indicating its potentials in NSCLC. Yet, several aspects regarding the application of CAR-T in solid tumors should be noticed. First, the "off-target" effect is one of the major causes that lead to increased toxicity and less efficacy. In hematological malignancies, for example, the $\mathrm{B}$ cell acute lymphoblastic leukemia (B-ALL), well tolerance could be observed during the treatment of CD19-targeted CAR-T cells [221] due to the ubiquitous expression of CD19 on differentiated B cells instead of hematopoietic stem cells. On the contrary, target antigen in a solid tumor may be additionally expressed in other tissue or organs which may lead to unexpected treatment-related toxicity. Besides, it is much more difficult to select candidate antigen in solid tumors with higher antigen heterogeneity [222-224]. Second, microenvironment in solid tumors was relatively immunosuppressive-preferred compared to hematological malignancies leading to less efficient CAR-T therapy in solid tumors. Indeed, ongoing trials regarding solid tumors may further decipher the uncertainty in the future.

The concept of bispecific antibody for oncogene was based on the simultaneous activation of different pathways driving tumor proliferation and growth [225-227]. So far, the bispecific antibody for lung cancer is still under initial researches. It is indeed an encouraging agent for lung cancer in the future according to the preliminary results. The novel EGFR/cMet bispecific antibody (JNJ-61186372), a fully humanized IgG1 antibody, was first put up in 2016, and its preliminary results in human were reported in 2018 WCLC [228, 229]. Objective response was shown in various activating EGFR mutations including $\mathrm{T} 790 \mathrm{~m}$ and exon 20 insertion. It seemed like the two separate targets combined may broaden its antitumor activity compared to single-target inhibition. However, the efficacy and duration of
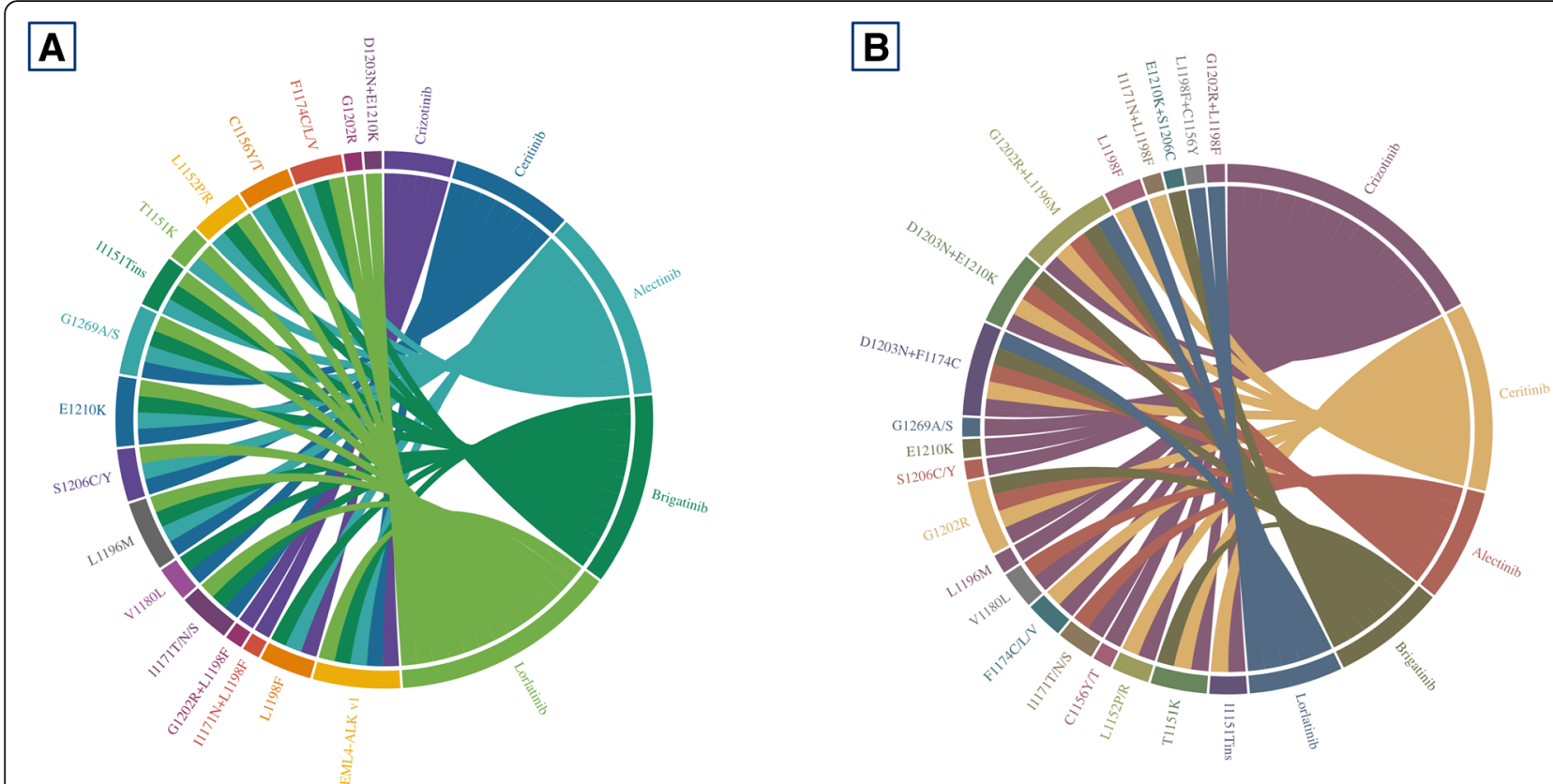

Fig. 2 Chord diagrams for sensitive and resistant mutations regarding ALK-TKIs. Both preclinical data and clinical reported cases (preferred) were enrolled to determine the efficacy of ALK-TKIs to different ALK-dependent mutations. Crizotinib had smallest sensitive mutation profiles compared to lorlatinib while opposite in resistant profiles. a Mutation profiles showed responsiveness to different ALK-TKIs. b Mutation profiles reported to be resistant to different ALK-TKIs 
response along with adverse events are warranted to further clarify its clinical application.

\section{Discussion}

Novel agents for lung cancer have been booming these years. Since the post era of IPASS and 2013 when immunotherapy has been crowned as one of the breakthroughs, remarkable clinical results from both tyrosine kinase inhibitors and checkpoint inhibitors for lung cancer have generated a great number of potential agents which significantly improved patients' survival beyond the era of chemotherapy. The current advances have been undoubtedly shifting the clinical paradigm for advanced lung cancer. So far, numerous potential agents including TKIs, CPIs, and underlying treatment modalities other than what we have mentioned above are under preclinical researches or early phase trials. Compared to previous standard treatment regarding TKIs, novel agents showed significant improvement in several aspects including improvement of $\mathrm{BBB}$ penetration, broadened target profiles, overcoming resistant mechanism, prolonged survival, and lower toxicity. In EGFR-TKIs, osimertinib has been undoubtedly claiming its essential clinical role in the first-line setting. However, whether sequential treatment following combination modalities (NEJ009 and JO25567 presented in 2018 ASCO meeting) or even novel second-generation TKI dacomitinib would be translated into better clinical outcome remained unknown. With potentially manageable acquired resistance, osimertinib would be temporally the best first option for untreated EGFR-mutant NSCLC patients. For ALK rearrangement, on the other hand, numerous highly potent targeted drugs were innovated and approved these years. Extremely complex mutational profiles were observed after the treatment of ALK-TKIs. Through chord diagram (Fig. 2) regarding sensitive and resistant mutational profiles of corresponding ALK-TKIs integrated from previous researches [106, 230-242], it is easy to tell that novel ALK-TKIs could cover more ALK-dependent mutations other than ALK fusion and meanwhile show antitumor activity in more resistant subtypes. Concurrent ALK-resistant mutations remained to be unyielding fields at the moment, and combination of different ALK-TKIs might be worthy to try in the future. For CPIs, numerous similar

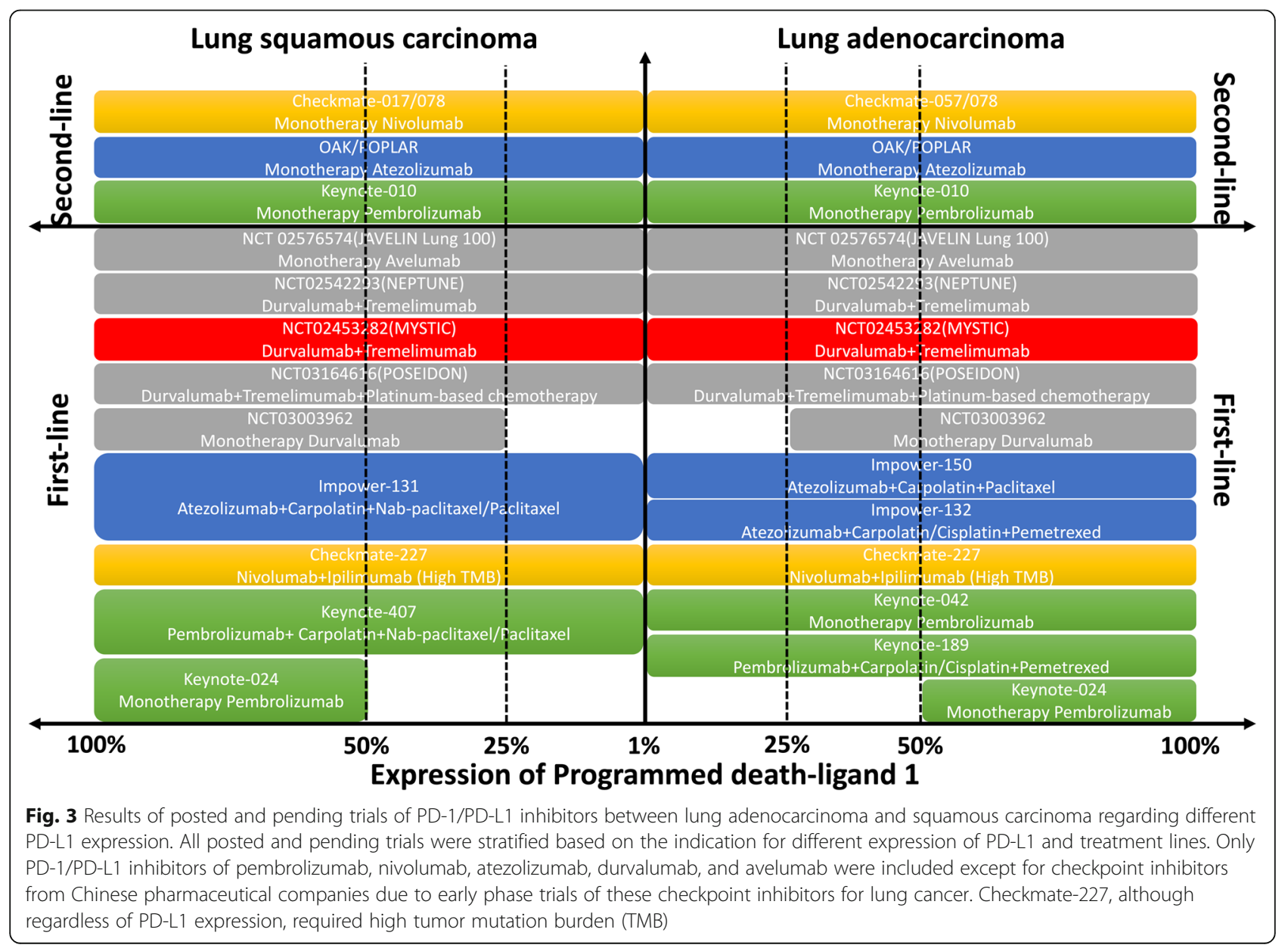




First-line Doublet CTIBeva
Fig. 4 Perspectives for the evolving modalities of immunotherapy in NSCLC. Treatment modalities involving immunotherapy in NSCLC had
evolved from second-line setting to first-line setting. Prior immunotherapy, highly selective patients, and combination strategies had raised
significant efficacy improvement but increased toxicities as well. Novel immunotherapy in the future combined with multiple novel biomarkers
may infinitely consolidate the clinical role of immunotherapy in advanced NSCLC

checkpoint inhibitors targeting PD-1/PD-L1 including CPIs developed by Chinese pharmaceutical companies [243] have been generated since the heat of immunotherapy. The clinical paradigm for wild-type NSCLC has been undoubtedly shifted with so many approved single or combination regimens involving CPIs in the first- and second-line setting. Some other phase III trials of novel checkpoint inhibitors were pending, and hopefully, more choices would be available in the first-line setting (Fig. 3). Indeed, due to first-mover advantage and limited understanding of how immunotherapy works within microenvironment, novel CPIs would be much tougher to compete as a single agent with previous CPIs. Combination treatment modality and clinical unmet needs, being the two major aspects, were ideal resolutions for the development of novel CPIs. To be noticed, challenges to immunotherapy remained to be unsolved including hyperprogression [244, 245], immune-related toxicities [246, 247], and primary/adaptive resistance to immunotherapy [248]. Moreover, potential novel treatment modalities have aroused great interest and their preliminary performances revealed remarkable prospects for development in lung cancer. Yet, most of the novel agents were still under the early stage of birth and further results should be expected. Whether these novel immunotherapy modalities may take place after the treatment failure of first-line checkpoint inhibitors in the future would be worth looking forward to (Fig. 4). At this moment, we are facing the condition of numerous novel agents developed for lung cancer. Improvement of clinical trials accelerating the application of novel drugs in clinical practice and discovery of novel effective targets along with much more precise biomarkers would be so much essential for anticancer treatment in the future.

\section{Abbreviations}

ADCC: Antibody-dependent cellular cytotoxicity; ALK: Anaplastic lymphoma kinase; B-ALL: B cell acute lymphoblastic leukemia; BBB: Blood-brain barrier; CAR-T: Chimeric antigen receptor T cell; CEA: Carcinoembryonic antigen; CNS: Central nerve system; CPI: Checkpoint inhibitor; DCR: Disease control rate; EGFR: Epidermal growth factor receptor; IgG1: Immunoglobulin G1; MSLN: Mesothelin; NSCLC: Non-small cell lung cancer; ORR: Objective response rate; OS: Overall survival; PD-1/PD-L1: Programmed cell death protein-1/programmed cell death protein ligand-1; PFS: Progression-free survival; PR: Partial response; RTK: Receptor tyrosine kinases; TAA: Tumorassociated antigen; TKl: Tyrosine kinase inhibitor

\section{Funding}

This work was supported by the National Natural Science Foundation (grant no.81673031 and 81872510, to WZ Zhong).

Availability of data and materials

All data generated or analyzed during this study are included in this published article.

\section{Authors' contributions}

WZZ and CZ designed the outline and drafted the manuscript. CZ designed the figures and tables. YLW and NBL offered professional suggestions to the manuscript. NBL provided the language editing. All authors read and approved the final manuscript.

Ethics approval and consent to participate

Not applicable

Consent for publication

Not applicable

\section{Competing interests}

The authors declare that they have no competing interests.

\section{Publisher's Note}

Springer Nature remains neutral with regard to jurisdictional claims in published maps and institutional affiliations.

\section{Author details}

'Guangdong Lung Cancer Institute, Guangdong Provincial Key Laboratory of Translational Medicine in Lung Cancer, Guangdong Provincial People's Hospital and Guangdong Academy of Medical Sciences, Guangzhou 510080, Guangdong, People's Republic of China. ${ }^{2}$ Princess Margaret Cancer Centre, 
Toronto, Ontario, Canada. ${ }^{3}$ School of Medicine, South China University of Technology, Guangzhou, People's Republic of China.

\section{Received: 6 February 2019 Accepted: 5 April 2019} Published online: 25 April 2019

\section{References}

1. Schiller $\mathrm{JH}$, et al. Comparison of four chemotherapy regimens for advanced non-small-cell lung cancer. N Engl J Med. 2002;346(2):92-8.

2. Sandler A, et al. Treatment outcomes by tumor histology in Eastern Cooperative Group Study E4599 of bevacizumab with paclitaxel/carboplatin for advanced non-small cell lung cancer. J Thorac Oncol. 2010;5(9):1416-23.

3. Herbst RS, Shin DM. Monoclonal antibodies to target epidermal growth factor receptor-positive tumors: a new paradigm for cancer therapy. Cancer. 2002;94(5):1593-611.

4. Leighl NB, et al. Randomized phase III study of matrix metalloproteinase inhibitor BMS-275291 in combination with paclitaxel and carboplatin in advanced non-small-cell lung cancer: National Cancer Institute of CanadaClinical Trials Group Study BR.18. J Clin Oncol. 2005;23(12):2831-9.

5. Paz-Ares $L$, et al. Phase III study of gemcitabine and cisplatin with or without aprinocarsen, a protein kinase C-alpha antisense oligonucleotide, in patients with advanced-stage non-small-cell lung cancer. J Clin Oncol. 2006; 24(9):1428-34.

6. Lynch TJ, et al. Activating mutations in the epidermal growth factor receptor underlying responsiveness of non-small-cell lung cancer to gefitinib. N Engl J Med. 2004;350(21):2129-39.

7. Maemondo M, et al. Gefitinib or chemotherapy for non-small-cell lung cancer with mutated EGFR. N Engl J Med. 2010;362(25):2380-8.

8. Mitsudomi T, et al. Gefitinib versus cisplatin plus docetaxel in patients with non-small-cell lung cancer harbouring mutations of the epidermal growth factor receptor (WJTOG3405): an open label, randomised phase 3 trial. Lancet Oncol. 2010;11(2):121-8.

9. Fukuoka $\mathrm{M}$, et al. Biomarker analyses and final overall survival results from a phase III, randomized, open-label, first-line study of gefitinib versus carboplatin/paclitaxel in clinically selected patients with advanced nonsmall-cell lung cancer in Asia (IPASS). J Clin Oncol. 2011;29(21):2866-74.

10. Han JY, et al. First-SIGNAL: first-line single-agent iressa versus gemcitabine and cisplatin trial in never-smokers with adenocarcinoma of the lung. J Clin Oncol. 2012;30(10):1122-8.

11. Wu YL, et al. First-line erlotinib versus gemcitabine/cisplatin in patients with advanced EGFR mutation-positive non-small-cell lung cancer: analyses from the phase III, randomized, open-label, ENSURE study. Ann Oncol. 2015;26(9): $1883-9$.

12. Sharma SV, et al. Epidermal growth factor receptor mutations in lung cancer. Nat Rev Cancer. 2007:7(3):169-81.

13. Lee CK, et al. Impact of EGFR inhibitor in non-small cell lung cancer on progression-free and overall survival: a meta-analysis. J Natl Cancer Inst. 2013:105(9):595-605.

14. Haaland $B$, et al. Meta-analysis of first-line therapies in advanced non-smallcell lung cancer harboring EGFR-activating mutations. J Thorac Oncol. 2014; 9(6):805-11.

15. Oxnard GR, et al. Acquired resistance to EGFR tyrosine kinase inhibitors in EGFR-mutant lung cancer: distinct natural history of patients with tumors harboring the T790M mutation. Clin Cancer Res. 2011;17(6):1616-22.

16. Zhou C, et al. Erlotinib versus chemotherapy as first-line treatment for patients with advanced EGFR mutation-positive non-small-cell lung cancer (OPTIMAL, CTONG-0802): a multicentre, open-label, randomised, phase 3 study. Lancet Oncol. 2011;12(8):735-42.

17. Sequist LV, et al. Phase III study of afatinib or cisplatin plus pemetrexed in patients with metastatic lung adenocarcinoma with EGFR mutations. J Clin Oncol. 2013;31(27):3327-34.

18. Wong DW, et al. The EML4-ALK fusion gene is involved in various histologic types of lung cancers from nonsmokers with wild-type EGFR and KRAS. Cancer. 2009;115(8):1723-33.

19. Gainor JF, et al. ALK rearrangements are mutually exclusive with mutations in EGFR or KRAS: an analysis of 1,683 patients with non-small cell lung cancer. Clin Cancer Res. 2013;19(15):4273-81.

20. Yu HA, et al. Analysis of tumor specimens at the time of acquired resistance to EGFR-TKI therapy in 155 patients with EGFR-mutant lung cancers. Clin Cancer Res. 2013;19(8):2240-7.
21. Tsao AS, et al. Scientific advances in lung cancer 2015. J Thorac Oncol. 2016; 11(5):613-38.

22. Couzin-Frankel J. Breakthrough of the year 2013. Cancer immunotherapy. Science. 2013;342(6165):1432-3.

23. Borghaei $\mathrm{H}$, et al. Nivolumab versus docetaxel in advanced nonsquamous non-small-cell lung cancer. N Engl J Med. 2015;373(17):1627-39.

24. Brahmer J, et al. Nivolumab versus docetaxel in advanced squamous-cell non-small-cell lung cancer. N Engl J Med. 2015;373(2):123-35.

25. Fehrenbacher $L$, et al. Atezolizumab versus docetaxel for patients with previously treated non-small-cell lung cancer (POPLAR): a multicentre, openlabel, phase 2 randomised controlled trial. Lancet. 2016;387(10030):1837-46.

26. Herbst RS, et al. Pembrolizumab versus docetaxel for previously treated, PDL1-positive, advanced non-small-cell lung cancer (KEYNOTE-010): a randomised controlled trial. Lancet. 2016;387(10027):1540-50.

27. Rittmeyer $\mathrm{A}$, et al. Atezolizumab versus docetaxel in patients with previously treated non-small-cell lung cancer (OAK): a phase 3, open-label, multicentre randomised controlled trial. Lancet. 2017;389(10066):255-65.

28. Reck M, et al. Pembrolizumab versus chemotherapy for PD-L1-positive nonsmall-cell lung cancer. N Engl J Med. 2016;375(19):1823-33.

29. Carbone DP, et al. First-line nivolumab in stage IV or recurrent non-smallcell lung cancer. N Engl J Med. 2017;376(25):2415-26.

30. Gettinger S, et al. Five-year follow-up of nivolumab in previously treated advanced non-small-cell lung cancer: results from the CA209-003 study. J Clin Oncol. 2018;36(17):1675-84.

31. von Pawel J, et al. Long-term survival in patients with advanced non-smallcell lung cancer treated with atezolizumab versus docetaxel: results from the randomised phase III OAK study. Eur J Cancer. 2019;107:124-32.

32. Huang $\mathrm{YH}$, et al. CEACAM1 regulates TIM-3-mediated tolerance and exhaustion. Nature. 2015;517(7534):386-90.

33. Ribas A. Adaptive immune resistance: how cancer protects from immune attack. Cancer Discov. 2015;5(9):915-9.

34. Champiat $\mathrm{S}$, et al. Hyperprogressive disease is a new pattern of progression in cancer patients treated by anti-PD-1/PD-L1. Clin Cancer Res. 2017;23(8):1920-8.

35. Ledford H. Promising cancer drugs may speed tumours in some patients. Nature. 2017;544(7648):13-4.

36. Engelman JA, et al. PF00299804, an irreversible pan-ERBB inhibitor, is effective in lung cancer models with EGFR and ERBB2 mutations that are resistant to gefitinib. Cancer Res. 2007;67(24):11924-32.

37. Gonzales AJ, et al. Antitumor activity and pharmacokinetic properties of PF00299804, a second-generation irreversible pan-erbB receptor tyrosine kinase inhibitor. Mol Cancer Ther. 2008;7(7):1880-9.

38. Wu YL, et al. Dacomitinib versus gefitinib as first-line treatment for patients with EGFR-mutation-positive non-small-cell lung cancer (ARCHER 1050): a randomised, open-label, phase 3 trial. Lancet Oncol. 2017;18(11):1454-66.

39. Yu JY, et al. Clinical outcomes of EGFR-TKI treatment and genetic heterogeneity in lung adenocarcinoma patients with EGFR mutations on exons 19 and 21. Chin J Cancer. 2016:35:30.

40. Yang W, et al. Postoperative survival of EGFR-TKI-targeted therapy in nonsmall cell lung cancer patients with EGFR 19 or 21 mutations: a retrospective study. World J Surg Oncol. 2017;15(1):197.

41. Mok TS, et al. Improvement in overall survival in a randomized study that compared dacomitinib with gefitinib in patients with advanced non-small-cell lung cancer and EGFR-activating mutations. J Clin Oncol. 2018:36(22):2244-50.

42. Mok T, et al. MA26.11 effects of dose modifications on the safety and efficacy of dacomitinib for EGFR mutation-positive NSCLC. J Thorac Oncol. 2018;13(10):S454.

43. Cross DA, et al. AZD9291, an irreversible EGFR TKI, overcomes T790Mmediated resistance to EGFR inhibitors in lung cancer. Cancer Discov. 2014; 4(9):1046-61.

44. Goss G, et al. Osimertinib for pretreated EGFR Thr790Met-positive advanced non-small-cell lung cancer (AURA2): a multicentre, open-label, single-arm, phase 2 study. Lancet Oncol. 2016;17(12):1643-52.

45. Yang JC, et al. Osimertinib in pretreated T790M-positive advanced nonsmall-cell lung cancer: AURA study phase II extension Component. J Clin Oncol. 2017;35(12):1288-96.

46. Soria JC, et al. Osimertinib in untreated EGFR-mutated advanced non-smallcell lung cancer. N Engl J Med. 2018;378(2):113-25.

47. Planchard D, et al. Postprogression outcomes for osimertinib versus standard-of-care EGFR-TKI in patients with previously untreated EGFRmutated advanced non-small cell lung cancer. Clin Cancer Res. 2019;25(7): 2058-63. 
48. Le $\mathrm{X}$, et al. Landscape of EGFR-dependent and -independent resistance mechanisms to osimertinib and continuation therapy beyond progression in EGFR-mutant NSCLC. Clin Cancer Res. 2018;24(24):6195-203.

49. Lin CC, et al. Outcomes in patients with non-small-cell lung cancer and acquired Thr790Met mutation treated with osimertinib: a genomic study. Lancet Respir Med. 2018;6(2):107-16.

50. Oxnard GR, et al. Assessment of resistance mechanisms and clinical implications in patients with EGFR T790M-positive lung cancer and acquired resistance to osimertinib. JAMA Oncol. 2018;4(11):1527-34.

51. Ramalingam SS, et al. Osimertinib as first-line treatment of EGFR mutationpositive advanced non-small-cell lung cancer. J Clin Oncol. 2018;36(9):841-9.

52. Zhang $P$, et al. Combined therapy with osimertinib and afatinib in a lung adenocarcinoma patient with EGFR T790M mutation and multiple HER2 alterations after resistance to icotinib: a case report. Thorac Cancer. 2018;9(12):1774-7.

53. York ER, et al. Tolerable and effective combination of full-dose crizotinib and osimertinib targeting met amplification sequentially emerging after T790M positivity in EGFR-mutant non-small cell lung cancer. J Thorac Oncol. 2017;12(7):e85-8.

54. Uchibori K, et al. Brigatinib combined with anti-EGFR antibody overcomes osimertinib resistance in EGFR-mutated non-small-cell lung cancer. Nat Commun. 2017:8:14768.

55. Shi $P$, et al. Overcoming acquired resistance to AZD9291, a third-generation EGFR inhibitor, through modulation of MEK/ERK-dependent Bim and Mcl-1 degradation. Clin Cancer Res. 2017;23(21):6567-79.

56. Piotrowska Z, et al. Landscape of acquired resistance to osimertinib in EGFRmutant nsclc and clinical validation of combined EGFR and RET inhibition with osimertinib and BLU-667 for acquired ret fusion. Cancer Discov. 2018;8(12):1529-39.

57. Nakatani $\mathrm{K}$, et al. KRAS and EGFR amplifications mediate resistance to rociletinib and osimertinib in acquired afatinib-resistant NSCLC harboring exon 19 deletion/T790M in EGFR. Mol Cancer Ther. 2019;18(1):112-26.

58. Martinez-Marti A, et al. Dual MET and ERBB inhibition overcomes intratumor plasticity in osimertinib-resistant-advanced non-small-cell lung cancer (NSCLC). Ann Oncol. 2017;28(10):2451-7.

59. Li L, et al. Transformation to small-cell carcinoma as an acquired resistance mechanism to AZD9291: a case report. Oncotarget. 2017:8(11):18609-14.

60. La Monica S, et al. Trastuzumab emtansine delays and overcomes resistance to the third-generation EGFR-TKI osimertinib in NSCLC EGFR mutated cell lines. J Exp Clin Cancer Res. 2017;36(1):174.

61. Jia Y, et al. Overcoming EGFR(T790M) and EGFR(C797S) resistance with mutant-selective allosteric inhibitors. Nature. 2016;534(7605):129-32.

62. Ho CC, et al. Acquired BRAF V600E mutation as resistant mechanism after treatment with osimertinib. J Thorac Oncol. 2017;12(3):567-72.

63. Arulananda S, et al. Combination osimertinib and gefitinib in C797S and T790M EGFR-mutated non-small cell lung cancer. J Thorac Oncol. 2017; 12(11):1728-32.

64. Umemura S, et al. Clinical outcome in patients with leptomeningeal metastasis from non-small cell lung cancer: Okayama Lung Cancer Study Group. Lung Cancer. 2012;77(1):134-9.

65. Palma JA, et al. Leptomeningeal carcinomatosis: prognostic value of clinical, cerebrospinal fluid, and neuroimaging features. Clin Neurol Neurosurg. 2013;115(1):19-25

66. Liao BC, et al. Epidermal growth factor receptor tyrosine kinase inhibitors for non-small-cell lung cancer patients with leptomeningeal carcinomatosis. J Thorac Oncol. 2015;10(12):1754-61.

67. Ballard $P$, et al. Preclinical comparison of osimertinib with other EGFR-TKIs in EGFR-mutant NSCLC brain metastases models, and early evidence of clinical brain metastases activity. Clin Cancer Res. 2016;22(20):5130-40.

68. Nanjo S, et al. Standard-dose osimertinib for refractory leptomeningeal metastases in T790M-positive EGFR-mutant non-small cell lung cancer. Br J Cancer. 2018;118(1):32-7.

69. Ahn MJ, et al. Activity and safety of AZD3759 in EGFR-mutant nonsmall-cell lung cancer with CNS metastases (BLOOM): a phase 1, openlabel, dose-escalation and dose-expansion study. Lancet Respir Med. 2017;5(11):891-902.

70. Rangachari $D$, et al. Brain metastases in patients with EGFR-mutated or ALK-rearranged non-small-cell lung cancers. Lung Cancer. 2015;88(1): 108-11.

71. Zeng Q, et al. Discovery and evaluation of clinical candidate AZD3759, a potent, oral active, central nervous system-penetrant, epidermal growth factor receptor tyrosine kinase inhibitor. J Med Chem. 2015; 58(20):8200-15.
72. Yang Z, et al. AZD3759, a BBB-penetrating EGFR inhibitor for the treatment of EGFR mutant NSCLC with CNS metastases. Sci Transl Med. 2016;8(368): 368ra172.

73. Mitsudomi T, Yatabe Y. Mutations of the epidermal growth factor receptor gene and related genes as determinants of epidermal growth factor receptor tyrosine kinase inhibitors sensitivity in lung cancer. Cancer Sci. 2007;98(12):1817-24.

74. Oxnard GR, et al. Natural history and molecular characteristics of lung cancers harboring EGFR exon 20 insertions. J Thorac Oncol. 2013;8(2):179-84.

75. Yasuda $\mathrm{H}$, et al. Structural, biochemical, and clinical characterization of epidermal growth factor receptor (EGFR) exon 20 insertion mutations in lung cancer. Sci Transl Med. 2013;5(216):216ra177.

76. Yasuda H, Kobayashi S, Costa DB. EGFR exon 20 insertion mutations in nonsmall-cell lung cancer: preclinical data and clinical implications. Lancet Oncol. 2012:13(1):e23-31.

77. Arcila ME, et al. EGFR exon 20 insertion mutations in lung adenocarcinomas: prevalence, molecular heterogeneity, and clinicopathologic characteristics. Mol Cancer Ther. 2013;12(2):220-9.

78. Robichaux JP, et al. Mechanisms and clinical activity of an EGFR and HER2 exon 20-selective kinase inhibitor in non-small cell lung cancer. Nat Med. 2018:24(5):638-46.

79. Heymach J, et al. OA02.06 a phase II trial of poziotinib in EGFR and HER2 exon 20 mutant non-small cell lung cancer (NSCLC). J Thorac Oncol. 2018; 13(10):S323-4

80. Neal J, et al. P1.13-44 safety, pk, and preliminary antitumor activity of the oral EGFR/HER2 exon 20 inhibitor TAK-788 in NSCLC. J Thorac Oncol. 2018; 13(10):S599.

81. Peters $\mathrm{S}$, et al. Activity of afatinib in heavily pretreated patients with ERBB2 mutation-positive advanced nsclc: findings from a global named patient use program. J Thorac Oncol. 2018;13(12):1897-905.

82. Mazieres J, et al. Lung cancer that harbors an HER2 mutation: epidemiologic characteristics and therapeutic perspectives. J Clin Oncol. 2013;31(16):1997-2003.

83. Goss GD, et al. Association of ERBB mutations with clinical outcomes of afatinib- or erlotinib-treated patients with lung squamous cell carcinoma: secondary analysis of the LUX-lung 8 randomized clinical trial. JAMA Oncol. 2018;4(9):1189-97.

84. De Greve J, et al. Clinical activity of afatinib (BIBW 2992) in patients with lung adenocarcinoma with mutations in the kinase domain of HER2/neu. Lung Cancer. 2012;76(1):123-7.

85. Dziadziuszko R, et al. Afatinib in non-small cell lung cancer with HER2 mutations: results of the prospective, open-label phase II NICHE trial of European Thoracic Oncology Platform (ETOP). J Thorac Oncol. 2019. https:// doi.org/10.1016/j.jtho.2019.02.017.

86. Ma F, et al. Phase I study and biomarker analysis of pyrotinib, a novel irreversible pan-ErbB receptor tyrosine kinase inhibitor, in patients with human epidermal growth factor receptor 2-positive metastatic breast cancer. J Clin Oncol. 2017;35(27):3105-12.

87. Li X, et al. Discovery and development of pyrotinib: a novel irreversible EGFR/HER2 dual tyrosine kinase inhibitor with favorable safety profiles for the treatment of breast cancer. Eur J Pharm Sci. 2017;110:51-61.

88. Wang $Y$, et al. HER2 exon 20 insertions in non-small cell lung cancer are sensitive to the irreversible pan-HER receptor tyrosine kinase inhibitor pyrotinib. Ann Oncol. 2018;30(3):447-55.

89. Solomon BJ, et al. First-line crizotinib versus chemotherapy in ALK-positive lung cancer. N Engl J Med. 2014;371(23):2167-77.

90. Katayama $R$, et al. Mechanisms of acquired crizotinib resistance in ALKrearranged lung cancers. Sci Transl Med. 2012;4(120):120ra17.

91. Doebele RC, et al. Mechanisms of resistance to crizotinib in patients with ALK gene rearranged non-small cell lung cancer. Clin Cancer Res. 2012; 18(5):1472-82.

92. Marsilje TH, et al. Synthesis, structure-activity relationships, and in vivo efficacy of the novel potent and selective anaplastic lymphoma kinase (ALK) inhibitor 5-chloro-N2-(2-isopropoxy-5-methyl-4-(piperidin-4-yl)phenyl)-N4-(2(isopropylsulf onyl)phenyl)pyrimidine-2,4-diamine (LDK378) currently in phase 1 and phase 2 clinical trials. J Med Chem. 2013;56(14):5675-90.

93. Shaw AT, et al. Ceritinib in ALK-rearranged non-small-cell lung cancer. N Engl J Med. 2014;370(13):1189-97.

94. Khozin S, et al. FDA approval: ceritinib for the treatment of metastatic anaplastic lymphoma kinase-positive non-small cell lung cancer. Clin Cancer Res. 2015;21(11):2436-9. 
95. Kim DW, et al. Activity and safety of ceritinib in patients with ALKrearranged non-small-cell lung cancer (ASCEND-1): updated results from the multicentre, open-label, phase 1 trial. Lancet Oncol. 2016;17(4):452-63.

96. Crino $L$, et al. Multicenter phase II study of whole-body and intracranial activity with ceritinib in patients with ALK-rearranged non-small-cell lung cancer previously treated with chemotherapy and crizotinib: results from ASCEND-2. J Clin Oncol. 2016;34(24):2866-73.

97. Soria JC, et al. First-line ceritinib versus platinum-based chemotherapy in advanced ALK-rearranged non-small-cell lung cancer (ASCEND-4): a randomised, open-label, phase 3 study. Lancet. 2017;389(10072):917-29.

98. Cho BC, et al. ASCEND-8: a randomized phase 1 study of ceritinib, $450 \mathrm{mg}$ or 600 mg, taken with a low-fat meal versus $750 \mathrm{mg}$ in fasted state in patients with anaplastic lymphoma kinase (ALK)-rearranged metastatic nonsmall cell lung cancer (NSCLC). J Thorac Oncol. 2017;12(9):1357-67.

99. Cho BC, et al. Efficacy and safety of ceritinib (450 mg/day or $600 \mathrm{mg} /$ day) with food vs $750 \mathrm{mg} /$ day fasted in patients with ALK-positive NSCLC: primary efficacy results from ASCEND-8 study. J Thorac Oncol. 2019. https:// doi.org/10.1016/j.jtho.2019.03.002.

100. Huber KV, et al. Stereospecific targeting of MTH1 by (S)-crizotinib as an anticancer strategy. Nature. 2014;508(7495):222-7.

101. Shaw AT, et al. Crizotinib in ROS1-rearranged non-small-cell lung cancer. $N$ Engl J Med. 2014;371(21):1963-71.

102. Wu YL, et al. Phase II study of crizotinib in east asian patients with ROS1-positive advanced non-small-cell lung cancer. J Clin Oncol. 2018;36(14):1405-11.

103. Lim SM, et al. Open-Label, multicenter, phase II study of ceritinib in patients with non-small-cell lung cancer harboring ROS1 rearrangement. J Clin Oncol. 2017:35(23):2613-8.

104. Zhang I, et al. Targeting brain metastases in ALK-rearranged non-small-cell lung cancer. Lancet Oncol. 2015;16(13):e510-21.

105. Awad MM, Shaw AT. ALK inhibitors in non-small cell lung cancer: crizotinib and beyond. Clin Adv Hematol Oncol. 2014;12(7):429-39.

106. Gainor JF, et al. Molecular mechanisms of resistance to first- and second generation ALK inhibitors in ALK-rearranged lung cancer. Cancer Discov. 2016;6(10):1118-33.

107. Katayama R, Lovly CM, Shaw AT. Therapeutic targeting of anaplastic lymphoma kinase in lung cancer: a paradigm for precision cancer medicine. Clin Cancer Res. 2015;21(10):2227-35.

108. Seto T, et al. CH5424802 (RO5424802) for patients with ALK-rearranged advanced non-small-cell lung cancer (AF-001.JP study): a single-arm, openlabel, phase 1-2 study. Lancet Oncol. 2013;14(7):590-8.

109. Hida T, et al. Alectinib versus crizotinib in patients with ALK-positive nonsmall-cell lung cancer (J-ALEX): an open-label, randomised phase 3 trial. Lancet. 2017;390(10089):29-39.

110. Peters $S$, et al. Alectinib versus crizotinib in untreated ALK-positive nonsmall-cell lung cancer. N Engl J Med. 2017;377(9):829-38.

111. Novello S, et al. Alectinib versus chemotherapy in crizotinib-pretreated anaplastic lymphoma kinase (ALK)-positive non-small-cell lung cancer: results from the phase III ALUR study. Ann Oncol. 2018;29(6):1409-16.

112. Kodama T, et al. Antitumor activity of the selective ALK inhibitor alectinib in models of intracranial metastases. Cancer Chemother Pharmacol. 2014;74(5): 1023-8.

113. Sakamoto H, et al. CH5424802, a selective ALK inhibitor capable of blocking the resistant gatekeeper mutant. Cancer Cell. 2011;19(5):679-90.

114. Costa DB, et al. CSF concentration of the anaplastic lymphoma kinase inhibitor crizotinib. J Clin Oncol. 2011:29(15):e443-5.

115. Lin JJ, et al. Efficacy of alectinib in patients with ALK-positive NSCLC and symptomatic or large CNS metastases. J Thorac Oncol. 2019;14(4):683-90.

116. Choi $Y L$, et al. EML4-ALK mutations in lung cancer that confer resistance to ALK inhibitors. N Engl J Med. 2010;363(18):1734-9.

117. Heuckmann JM, et al. ALK mutations conferring differential resistance to structurally diverse ALK inhibitors. Clin Cancer Res. 2011;17(23):7394-401.

118. Friboulet $\mathrm{L}$, et al. The ALK inhibitor ceritinib overcomes crizotinib resistance in non-small cell lung cancer. Cancer Discov. 2014;4(6):662-73.

119. Ignatius Ou SH, et al. Next-generation sequencing reveals a Novel NSCLC ALK F1174V mutation and confirms ALK G1202R mutation confers highlevel resistance to alectinib (CH5424802/RO5424802) in ALK-rearranged NSCLC patients who progressed on crizotinib. J Thorac Oncol. 2014;9(4): 549-53.

120. Huang WS, et al. Discovery of brigatinib (AP26113), a phosphine oxidecontaining, potent, orally active inhibitor of anaplastic lymphoma kinase. J Med Chem. 2016;59(10):4948-64.
121. Zhang S, et al. The potent ALK inhibitor brigatinib (AP26113) overcomes mechanisms of resistance to first- and second-generation ALK inhibitors in preclinical models. Clin Cancer Res. 2016;22(22):5527-38.

122. Lin JJ, et al. Brigatinib in patients with alectinib-refractory ALK-positive NSCLC. J Thorac Oncol. 2018;13(10):1530-8.

123. Kim DW, et al. Brigatinib in patients with crizotinib-refractory anaplastic lymphoma kinase-positive non-small-cell lung cancer: a randomized, multicenter phase II trial. J Clin Oncol. 2017;35(22):2490-8.

124. Camidge DR, et al. Brigatinib versus crizotinib in ALK-positive non-small-cell lung cancer. N Engl J Med. 2018;379(21):2027-39.

125. Zhou J, et al. Crizotinib in patients with anaplastic lymphoma kinasepositive advanced non-small cell lung cancer versus chemotherapy as a first-line treatment. BMC Cancer. 2018;18(1):10.

126. Nishio M, et al. Crizotinib versus chemotherapy in asian patients with ALKpositive advanced non-small cell lung cancer. Cancer Res Treat. 2018;50(3): 691-700.

127. Shaw AT, et al. Crizotinib versus chemotherapy in advanced ALK-positive lung cancer. N Engl J Med. 2013;368(25):2385-94.

128. Shaw AT, et al. Ceritinib versus chemotherapy in patients with ALKrearranged non-small-cell lung cancer previously given chemotherapy and crizotinib (ASCEND-5): a randomised, controlled, open-label, phase 3 trial. Lancet Oncol. 2017;18(7):874-86.

129. Johnson TW, et al. Discovery of (10R)-7-amino-12-fluoro-2,10,16-trimethyl-15oxo-10,15,16,17-tetrahydro-2H-8,4-(m etheno) pyrazolo $[4,3-h][2,5,11]$ benzoxadiazacyclotetradecine-3-carbonitrile (PF-06463922), a macrocyclic inhibitor of anaplastic lymphoma kinase (ALK) and c-ros oncogene 1 (ROS1) with preclinical brain exposure and broad-spectrum potency against ALKresistant mutations. J Med Chem. 2014:57(11):4720-44.

130. Redaelli S, et al. Lorlatinib treatment elicits multiple on- and off-target mechanisms of resistance in ALK-driven cancer. Cancer Res. 2018;78(24): 6866-80.

131. Solomon BJ, et al. Lorlatinib in patients with ALK-positive non-small-cell lung cancer: results from a global phase 2 study. Lancet Oncol. 2018;19(12): 1654-67.

132. Shaw AT, et al. Lorlatinib in non-small-cell lung cancer with ALK or ROS1 rearrangement: an international, multicentre, open-label, single-arm first-inman phase 1 trial. Lancet Oncol. 2017;18(12):1590-9.

133. Lovly CM, et al. Insights into ALK-driven cancers revealed through development of novel ALK tyrosine kinase inhibitors. Cancer Res. 2011; 71(14):4920-31.

134. Horn L, et al. MINI01.02: response and plasma genotyping from phase $1 / I$ trial of ensartinib (X-396) in patients (pts) with ALK+NSCLC: topic: medical oncology. J Thorac Oncol. 2016;11(11):S256-7.

135. Horn L, et al. Ensartinib (X-396) in ALK-positive non-small cell lung cancer: results from a first-in-human phase I/II, multicenter Study. Clin Cancer Res. 2018;24(12):2771-9.

136. Horn L, et al. P1.13-02 eXalt3: phase 3 randomized study comparing ensartinib to crizotinib in anaplastic lymphoma kinase positive non-small cell lung cancer patients. J Thorac Oncol. 2018;13(10):S582.

137. Stransky N, et al. The landscape of kinase fusions in cancer. Nat Commun. 2014;5:4846.

138. Vaishnavi A, et al. Oncogenic and drug-sensitive NTRK1 rearrangements in lung cancer. Nat Med. 2013;19(11):1469-72.

139. Drilon A, et al. Safety and antitumor activity of the multitargeted pan-TRK, ROS1, and ALK inhibitor entrectinib: combined results from two phase I trials (ALKA-372-001 and STARTRK-1). Cancer Discov. 2017;7(4):400-9.

140. Drilon A, et al. Efficacy of larotrectinib in TRK fusion-positive cancers in adults and children. N Engl J Med. 2018;378(8):731-9.

141. Drilon A, et al. What hides behind the MASC: clinical response and acquired resistance to entrectinib after ETV6-NTRK3 identification in a mammary analogue secretory carcinoma (MASC). Ann Oncol. 2016;27(5):920-6.

142. Russo $M$, et al. Acquired resistance to the TRK inhibitor entrectinib in colorectal cancer. Cancer Discov. 2016;6(1):36-44.

143. Drilon A, et al. A Next-generation TRK kinase inhibitor overcomes acquired resistance to prior TRK kinase inhibition in patients with TRK fusion-positive solid tumors. Cancer Discov. 2017;7(9):963-72.

144. Drilon A, et al. Repotrectinib (TPX-0005) is a next-generation ROS1/TRK/ALK inhibitor that potently inhibits ROS1/TRK/ALK solvent- front mutations. Cancer Discov. 2018;8(10):1227-36.

145. Mulligan LM. RET revisited: expanding the oncogenic portfolio. Nat Rev Cancer. 2014;14(3):173-86. 
146. Lee $\mathrm{SH}$, et al. Vandetanib in pretreated patients with advanced non-small cell lung cancer-harboring RET rearrangement: a phase II clinical trial. Ann Oncol. 2017;28(2):292-7.

147. Kato $S$, et al. RET aberrations in diverse cancers: next-generation sequencing of 4,871 Patients. Clin Cancer Res. 2017;23(8):1988-97.

148. Li GG, et al. Antitumor activity of RXDX-105 in multiple cancer types with RET rearrangements or mutations. Clin Cancer Res. 2017;23(12):2981-90.

149. Drilon A, et al. Cabozantinib in patients with advanced RET-rearranged nonsmall-cell lung cancer: an open-label, single-centre, phase 2, single-arm trial. Lancet Oncol. 2016;17(12):1653-60.

150. Drilon A, et al. A phase I/lb trial of the VEGFR-sparing multikinase RET inhibitor RXDX-105. Cancer Discov. 2019;9(3):384-95.

151. Subbiah $V$, et al. Selective RET kinase inhibition for patients with RET-altered cancers. Ann Oncol. 2018;29(8):1869-76.

152. Kurzrock R, et al. Activity of XL184 (Cabozantinib), an oral tyrosine kinase inhibitor, in patients with medullary thyroid cancer. J Clin Oncol. 2011; 29(19):2660-6.

153. Subbiah $V$, et al. Precision targeted therapy with BLU-667 for RET-driven cancers. Cancer Discov. 2018;8(7):836-49.

154. Liu X, et al. A novel kinase inhibitor, INCB28060, blocks C-MET-dependent signaling, neoplastic activities, and cross-talk with EGFR and HER-3. Clin Cancer Res. 2011;17(22):7127-38.

155. Frampton GM, et al. Activation of MET via diverse exon 14 splicing alterations occurs in multiple tumor types and confers clinical sensitivity to MET inhibitors. Cancer Discov. 2015;5(8):850-9.

156. Chen HJ, et al. Clinicopathologic and molecular features of epidermal growth factor receptor T790M mutation and c-MET amplification in tyrosine kinase inhibitor-resistant Chinese non-small cell lung cancer. Pathol Oncol Res. 2009;15(4):651-8.

157. Bean J, et al. MET amplification occurs with or without T790M mutations in EGFR mutant lung tumors with acquired resistance to gefitinib or erlotinib. Proc Natl Acad Sci U S A. 2007;104(52):20932-7.

158. Sequist LV, et al. Genotypic and histological evolution of lung cancers acquiring resistance to EGFR inhibitors. Sci Transl Med. 2011;3(75): 75 ra26.

159. Engelman JA, et al. MET amplification leads to gefitinib resistance in lung cancer by activating ERBB3 signaling. Science. 2007;316(5827):1039-43.

160. Lara MS, et al. Preclinical evaluation of MET Inhibitor INC-280 with or without the epidermal growth factor receptor inhibitor erlotinib in nonsmall-cell lung cancer. Clin Lung Cancer. 2017;18(3):281-5.

161. Wu YL, et al. Phase Ib/II study of capmatinib (INC280) plus gefitinib after failure of epidermal growth factor receptor (EGFR) inhibitor therapy in patients with EGFR-mutated, MET factor-dysregulated non-small-cell lung cancer. J Clin Oncol. 2018;36(31):3101-9.

162. Kim S, et al. Acquired resistance of MET-amplified non-small cell lung cancer cells to the MET inhibitor capmatinib. Cancer Res Treat. 2018. https://doi. org/10.4143/crt.2018.052.

163. Paik PK, et al. Clinical characteristics of patients with lung adenocarcinomas harboring BRAF mutations. J Clin Oncol. 2011;29(15):2046-51.

164. Kris MG, et al. Using multiplexed assays of oncogenic drivers in lung cancers to select targeted drugs. Jama. 2014;311(19):1998-2006.

165. Marchetti A, et al. Clinical features and outcome of patients with non-small-cell lung cancer harboring BRAF mutations. J Clin Oncol. 2011;29(26):3574-9.

166. Cardarella S, et al. Clinical, pathologic, and biologic features associated with BRAF mutations in non-small cell lung cancer. Clin Cancer Res. 2013;19(16): 4532-40.

167. Hyman DM, et al. Vemurafenib in multiple nonmelanoma cancers with BRAF V600 mutations. N Engl J Med. 2015;373(8):726-36.

168. King AJ, et al. Dabrafenib; preclinical characterization, increased efficacy when combined with trametinib, while BRAF/MEK tool combination reduced skin lesions. PLoS One. 2013;8(7):e67583.

169. Planchard D, et al. Dabrafenib in patients with BRAF(V600E)-positive advanced non-small-cell lung cancer: a single-arm, multicentre, open-label, phase 2 trial. Lancet Oncol. 2016;17(5):642-50.

170. Larkin J, et al. Combined vemurafenib and cobimetinib in BRAF-mutated melanoma. N Engl J Med. 2014;371(20):1867-76.

171. Flaherty KT, et al. Combined BRAF and MEK inhibition in melanoma with BRAF V600 mutations. N Engl J Med. 2012;367(18):1694-703.

172. Planchard D, et al. Dabrafenib plus trametinib in patients with previously untreated BRAF(V600E)-mutant metastatic non-small-cell lung cancer: an open-label, phase 2 trial. Lancet Oncol. 2017;18(10):1307-16.
173. Planchard D, et al. Dabrafenib plus trametinib in patients with previously treated BRAF(V600E)-mutant metastatic non-small cell lung cancer: an openlabel, multicentre phase 2 trial. Lancet Oncol. 2016;17(7):984-93.

174. Xie C, et al. Preclinical characterization of anlotinib, a highly potent and selective vascular endothelial growth factor receptor-2 inhibitor. Cancer Sci. 2018:109(4):1207-19.

175. Taurin S, et al. Endometrial cancers harboring mutated fibroblast growth factor receptor 2 protein are successfully treated with a new small tyrosine kinase inhibitor in an orthotopic mouse model. Int J Gynecol Cancer. 2018; 28(1):152-60.

176. Shen $\mathrm{G}$, et al. Anlotinib: a novel multi-targeting tyrosine kinase inhibitor in clinical development. J Hematol Oncol. 2018;11(1):120.

177. Lin $B$, et al. Anlotinib inhibits angiogenesis via suppressing the activation of VEGFR2, PDGFRbeta and FGFR1. Gene. 2018;654:77-86.

178. Han B, et al. Anlotinib as a third-line therapy in patients with refractory advanced non-small-cell lung cancer: a multicentre, randomised phase II trial (ALTER0302). Br J Cancer. 2018;118(5):654-61.

179. Diggs LP, Hsueh EC. Utility of PD-L1 immunohistochemistry assays for predicting PD-1/PD-L1 inhibitor response. Biomark Res. 2017:5:12.

180. Langer CJ, et al. Carboplatin and pemetrexed with or without pembrolizumab for advanced, non-squamous non-small-cell lung cancer: a randomised, phase 2 cohort of the open-label KEYNOTE-021 study. Lancet Oncol. 2016;17(11):1497-508.

181. Seto $T$, et al. Erlotinib alone or with bevacizumab as first-line therapy in patients with advanced non-squamous non-small-cell lung cancer harbouring EGFR mutations (JO25567): an open-label, randomised, multicentre, phase 2 study. Lancet Oncol. 2014;15(11):1236-44.

182. Rosell $R$, et al. Erlotinib and bevacizumab in patients with advanced nonsmall-cell lung cancer and activating EGFR mutations (BELIEF): an international, multicentre, single-arm, phase 2 trial. Lancet Respir Med. 2017; 5(5):435-44

183. Han B, et al. Combination of chemotherapy and gefitinib as first-line treatment for patients with advanced lung adenocarcinoma and sensitive EGFR mutations: a randomized controlled trial. Int J Cancer. 2017;141(6): 1249-56.

184. Paz-Ares $L$, et al. Pembrolizumab plus chemotherapy for squamous nonsmall-cell lung cancer. N Engl J Med. 2018;379(21):2040-51.

185. Gandhi L, et al. Pembrolizumab plus chemotherapy in metastatic non-smallcell lung cancer. N Engl J Med. 2018;378(22):2078-92.

186. Hellmann MD, et al. Nivolumab plus ipilimumab in lung cancer with a high tumor mutational burden. N Engl J Med. 2018;378(22):2093-104.

187. Socinski MA, et al. Atezolizumab for first-line treatment of metastatic nonsquamous NSCLC. N Engl J Med. 2018;378(24):2288-301.

188. Gulley $\mathrm{J}$, et al. Avelumab for patients with previously treated metastatic or recurrent non-small-cell lung cancer (JAVELIN Solid Tumor): dose-expansion cohort of a multicentre, open-label, phase 1b trial. Lancet Oncol. 2017;18(5): 599-610.

189. Fujii R, et al. Enhanced killing of chordoma cells by antibody-dependent cell-mediated cytotoxicity employing the novel anti-PD-L1 antibody avelumab. Oncotarget. 2016;7(23):33498-511.

190. Boyerinas B, et al. Antibody-dependent cellular cytotoxicity activity of a novel anti-PD-L1 antibody avelumab (MSB0010718C) on human tumor cells. Cancer Immunol Res. 2015:3(10):1148-57.

191. Barlesi F, et al. Avelumab versus docetaxel in patients with platinum-treated advanced non-small-cell lung cancer (JAVELIN Lung 200): an open-label, randomised, phase 3 study. Lancet Oncol. 2018;19(11):1468-79.

192. Stewart R, et al. Identification and characterization of MEDI4736, an antagonistic anti-PD-L1 monoclonal antibody. Cancer Immunol Res. 2015; 3(9):1052-62.

193. Postow MA, Callahan MK, Wolchok JD. Immune checkpoint blockade in cancer therapy. J Clin Oncol. 2015;33(17):1974-82.

194. Planchard D, et al. A phase III study of durvalumab (MED14736) with or without tremelimumab for previously treated patients with advanced NSCLC: rationale and protocol design of the ARCTIC study. Clin Lung Cancer. 2016;17(3):232-236.e1.

195. Antonia S, et al. Safety and antitumour activity of durvalumab plus tremelimumab in non-small cell lung cancer: a multicentre, phase $1 \mathrm{~b}$ study. Lancet Oncol. 2016:17(3):299-308.

196. Garassino MC, et al. Durvalumab as third-line or later treatment for advanced non-small-cell lung cancer (ATLANTIC): an open-label, single-arm, phase 2 study. Lancet Oncol. 2018;19(4):521-36. 
197. Antonia SJ, et al. Durvalumab after chemoradiotherapy in stage III nonsmall-cell lung cancer. N Engl J Med. 2017;377(20):1919-29.

198. Antonia SJ, et al. Overall survival with durvalumab after chemoradiotherapy in stage III NSCLC. N Engl J Med. 2018;379(24):2342-50.

199. Rosenberg SA, Restifo NP. Adoptive cell transfer as personalized immunotherapy for human cancer. Science. 2015;348(6230):62-8.

200. Marin-Acevedo JA, et al. Cancer immunotherapy beyond immune checkpoint inhibitors. J Hematol Oncol. 2018;11(1):8.

201. Ott PA, Hodi FS, Robert C. CTLA-4 and PD-1/PD-L1 blockade: new immunotherapeutic modalities with durable clinical benefit in melanoma patients. Clin Cancer Res. 2013;19(19):5300-9.

202. lafolla MAJ, Juergens RA. Update on programmed death-1 and programmed death-ligand 1 inhibition in the treatment of advanced or metastatic non-small cell lung cancer. Front Oncol. 2017;7:67.

203. Chen L, Flies DB. Molecular mechanisms of T cell co-stimulation and coinhibition. Nat Rev Immunol. 2013;13(4):227-42.

204. Johnson LA, et al. Rational development and characterization of humanized anti-EGFR variant III chimeric antigen receptor T cells for glioblastoma. Sci Transl Med. 2015;7(275):275ra22.

205. Beatty GL, et al. Mesothelin-specific chimeric antigen receptor mRNAengineered $T$ cells induce anti-tumor activity in solid malignancies. Cancer Immunol Res. 2014;2(2):112-20.

206. Adusumilli PS, et al. Regional delivery of mesothelin-targeted CAR T cell therapy generates potent and long-lasting CD4-dependent tumor immunity. Sci Transl Med. 2014;6(261):261ra151.

207. Topalian SL, et al. Safety, activity, and immune correlates of anti-PD-1 antibody in cancer. N Engl J Med. 2012;366(26):2443-54.

208. Schmid P, et al. Atezolizumab and nab-paclitaxel in advanced triplenegative breast cancer. N Engl J Med. 2018;379(22):2108-21.

209. Schachter J, et al. Pembrolizumab versus ipilimumab for advanced melanoma: final overall survival results of a multicentre, randomised, openlabel phase 3 study (KEYNOTE-006). Lancet. 2017;390(10105):1853-62.

210. Massard C, et al. Safety and efficacy of durvalumab (MEDI4736), an antiprogrammed cell death ligand-1 immune checkpoint inhibitor, in patients with advanced urothelial bladder cancer. J Clin Oncol. 2016;34(26):3119-25.

211. Garon EB, et al. Pembrolizumab for the treatment of non-small-cell lung cancer. N Engl J Med. 2015;372(21):2018-28.

212. Dirix LY, et al. Avelumab, an anti-PD-L1 antibody, in patients with locally advanced or metastatic breast cancer: a phase 1b JAVELIN Solid Tumor study. Breast Cancer Res Treat. 2018;167(3):671-86.

213. Brahmer JR, et al. Safety and activity of anti-PD-L1 antibody in patients with advanced cancer. N Engl J Med. 2012;366(26):2455-65.

214. Turtle CJ, et al. CD19 CAR-T cells of defined CD4+:CD8+ composition in adult B cell ALL patients. J Clin Invest. 2016;126(6):2123-38.

215. Park JH, et al. Long-term follow-up of CD19 CAR therapy in acute lymphoblastic leukemia. N Engl J Med. 2018;378(5):449-59.

216. Maude SL, et al. Chimeric antigen receptor T cells for sustained remissions in leukemia. N Engl J Med. 2014;371(16):1507-17.

217. Lee DW, et al. T cells expressing CD19 chimeric antigen receptors for acute lymphoblastic leukaemia in children and young adults: a phase 1 doseescalation trial. Lancet. 2015;385(9967):517-28.

218. Davila ML, et al. Efficacy and toxicity management of 19-28z CAR T cell therapy in B cell acute lymphoblastic leukemia. Sci Transl Med. 2014;6(224):224ra25.

219. Li J, et al. Chimeric antigen receptor T cell (CAR-T) immunotherapy for solid tumors: lessons learned and strategies for moving forward. J Hematol Oncol. 2018;11(1):22

220. Liu B, Song Y, Liu D. Clinical trials of CAR-T cells in China. J Hematol Oncol. 2017;10(1):166

221. Mueller KT, et al. Cellular kinetics of CTL019 in relapsed/refractory B-cell acute lymphoblastic leukemia and chronic lymphocytic leukemia. Blood. 2017;130(21):2317-25.

222. Marusyk A, Polyak K. Tumor heterogeneity: causes and consequences. Biochim Biophys Acta. 2010;1805(1):105-17.

223. Fidler IJ, Hart IR. Biological diversity in metastatic neoplasms: origins and implications. Science. 1982;217(4564):998-1003.

224. Feng K, et al. Chimeric antigen receptor-modified T cells for the immunotherapy of patients with EGFR-expressing advanced relapsed/ refractory non-small cell lung cancer. Sci China Life Sci. 2016;59(5):468-79.

225. Planchard D, et al. EGFR-independent mechanisms of acquired resistance to AZD9291 in EGFR T790M-positive NSCLC patients. Ann Oncol. 2015;26(10): 2073-8.
226. Pao W, et al. Acquired resistance of lung adenocarcinomas to gefitinib or erlotinib is associated with a second mutation in the EGFR kinase domain PLOS Med. 2005;2(3):e73.

227. Jiang SX, et al. EGFR genetic heterogeneity of nonsmall cell lung cancers contributing to acquired gefitinib resistance. Int J Cancer. 2008;123(11): 2480-6.

228. Park K, et al. OA10.06 a first-in-human phase 1 trial of the EGFR-CMET bispecific antibody JNJ-61186372 in patients with advanced non-small cell lung cancer (NSCLC). J Thorac Oncol. 2018;13(10):S344-5.

229. Moores SL, et al. A novel bispecific antibody targeting EGFR and cMet is effective against EGFR inhibitor-resistant lung tumors. Cancer Res. 2016; 76(13):3942-53.

230. Childress MA, et al. ALK fusion partners impact response to ALK inhibition: differential effects on sensitivity, cellular phenotypes, and biochemical properties. Mol Cancer Res. 2018;16(11):1724-36.

231. Esfahani K, Agulnik JS, Cohen V. A systemic review of resistance mechanisms and ongoing clinical trials in ALK-rearranged non-small cell lung cancer. Front Oncol. 2014;4:174.

232. Katayama $R$, et al. Two novel ALK mutations mediate acquired resistance to the next-generation ALK inhibitor alectinib. Clin Cancer Res. 2014;20(22): 5686-96.

233. Lin JJ, Riely GJ, Shaw AT. Targeting ALK: precision medicine takes on drug resistance. Cancer Discov. 2017;7(2):137-55.

234. Muller IB, et al. Overcoming crizotinib resistance in ALK-rearranged NSCLC with the second-generation ALK-inhibitor ceritinib. Expert Rev Anticancer Ther. 2016;16(2):147-57.

235. Okada K, et al. Prediction of ALK mutations mediating ALK-TKIs resistance and drug re-purposing to overcome the resistance. EBioMedicine. 2019;41: 105-19. https://doi.org/10.1016/j.ebiom.2019.01.019.

236. Ou SH, et al. 11171 missense mutation (particularly $11171 \mathrm{~N}$ ) is a common resistance mutation in ALK-positive NSCLC patients who have progressive disease while on alectinib and is sensitive to ceritinib. Lung Cancer. 2015; 88(2):231-4.

237. Ou SH, et al. ALK F1174V mutation confers sensitivity while ALK I1171 mutation confers resistance to alectinib. The importance of serial biopsy post progression. Lung Cancer. 2016;91:70-2.

238. Sabari JK, et al. The activity, safety, and evolving role of brigatinib in patients with ALK-rearranged non-small cell lung cancers. Onco Targets Ther. 2017;10:1983-92.

239. Yoda S, et al. Sequential ALK inhibitors can select for lorlatinib-resistant compound ALK mutations in ALK-positive lung cancer. Cancer Discov. 2018; $8(6): 714-29$

240. Zhu WW, et al. Identification of a novel T1151K ALK mutation in a patient with ALK-rearranged NSCLC with prior exposure to crizotinib and ceritinib. Lung Cancer. 2017;110:32-4.

241. Zhu WW, et al. Dramatic response to alectinib in a lung cancer patient with a novel VKORC1L1-ALK fusion and an acquired ALK T1151K mutation. Lung Cancer (Auckl). 2018;9:111-6.

242. Zou HY, et al. PF-06463922, an ALK/ROS1 inhibitor, overcomes resistance to first and second generation ALK inhibitors in preclinical models. Cancer Cell. 2015;28(1):70-81.

243. Liu SY, Wu YL. Ongoing clinical trials of PD-1 and PD-L1 inhibitors for lung cancer in China. J Hematol Oncol. 2017;10(1):136.

244. Fuentes-Antras J, Provencio M, Diaz-Rubio E. Hyperprogression as a distinct outcome after immunotherapy. Cancer Treat Rev. 2018;70:16-21.

245. Kurman JS, Murgu SD. Hyperprogressive disease in patients with non-small cell lung cancer on immunotherapy. J Thorac Dis. 2018;10(2):1124-8.

246. Johnson DB, et al. Fulminant myocarditis with combination immune checkpoint blockade. N Engl J Med. 2016;375(18):1749-55.

247. Kanai $\mathrm{O}$, et al. Concurrence of nivolumab-induced interstitial lung disease and cancer invasion. Respirol Case Rep. 2017;5(6):e00257.

248. Marin-Acevedo JA, et al. Next generation of immune checkpoint therapy in cancer: new developments and challenges. J Hematol Oncol. 2018;11(1):39. 